\title{
Nonlinear Feedback Guidance Law for Aero-Assisted Orbit Transfer Maneuvers
}

\author{
Final Report \\ Prepared for \\ NASA Langley Research Center \\ Hampton, VA 23665-5225 \\ Under Grant NAG 1-1243
}

March 1992

Principal Investigator : Dr. P. K. A. Menon

NASA Technical Monitor : Dr. D. D. Moerder

Georgia Insticute of Technology

School of Aerospace Engineering

Atlanta, GA 30332-0150

(NASA-CR-190129) NONL INEAR FFEDBACK GUINANCE LAW FUR AERO-ASSISTED OREIT TRANSFER MANEUVEKS Final Technical Report (ceoraia Inst. of rech.) $31 \mathrm{p}$ CSCl $22 \mathrm{~A}$ 


\begin{abstract}
Aero-assisted orbit transfer vehicles have the potential for significantly reducing the fuel requirements in certain classes of orbit transfer operations. Development of a nonlinear feedback guidance law for performing aero-assisted maneuvers that accomplish simultaneous change of all the orbital elements with least vehicle acceleration magnitude is discussed. The analysis is based on a sixth-order nonlinear point-mass vehicle model with lift, bank angle, thrust and drag modulation as the control variables. The guidance law uses detailed vehicle aerodynamic and the atmosphere models in the feedback loop. Higher-order gravitational harmonics, planetary atmosphere rotation and ambient winds are included in the formulation. Due to modest computational requirements, the guidance law is implementable on-board an orbit transfer vehicle. The guidance law performance is illustrated for three sets of boundary conditions.
\end{abstract}

\title{
Introduction
}

The concept of using maneuvers in the planetary atmosphere for orbital plane change was first advanced by London [1]. This concept did not receive significant attention in the literature until the early eighties. Since then there has been significant research activity in evaluating the payoffs and in synthesizing guidance laws for the aero-assisted orbit transfer mission. For instance, References 2 and 3 examines the performance payoffs in employing such maneuvers. Guidance law development using various vehicle model approximations are discussed in References 4 - 10. A Lyapunov optimal feedback control law for the orbit transfer mission was proposed in Reference 11. Using an approximate guidance law, Reference 12 discusses the effect of earth rotation on the aeroassisted orbit transfer mission.

The aeroassisted orbit transfer maneuver guidance laws reported in the literature [2 -12] achieve a desired velocity vector orientation at the point of exit from the atmosphere while optimizing a specified performance index. The optimization objectives have included the minimization of energy loss during the atmospheric pass, minimum fuel, minimum aerodynamic heating, and minimum flight time. Minimum acceleration magnitude criterion is used as the performance index in the present research. This criterion will ensure that the emerging guidance law results in the least structural load on the vehicle, permitting implementation on-board the orbit transfer vehicle.

The present paper addresses the problem of simultaneously controlling all the six components of the vehicle state vector at the atmospheric exit. Such a formulation enables simultaneous in-plane and out-of-plane aeroassisted orbit transfer maneuvers together with a control of the flight time, latitude and longitude at the exit from the atmosphere. Control of flight time is useful for establishing the time-of-perigee passage or the argument of perigee of the resulting orbit, while the control of terminal latitude and longitude are important to assure adequate ground station visibility. 
The focus of the present research is on the development of a feedback guidance law for on-board implementation. Feedback linearization theory will be used to develop an aero-assisted orbit transfer guidance law that achieves a specified terminal state vector in a specified time period. This paper is an outgrowth of the preliminary research outlined in Reference 13.

The feedback linearization concept has been previously applied to various nonlinear guidance problems $[14,15]$ with significant success. The present research differs from the studies given in References $4-12$ in several ways. For instance, a planet centered Cartesian coordinate system is employed in the present formulation. The effects of planet oblateness, atmosphere rotation and ambient winds are included. Note that neglecting the atmosphere rotation will result in an overestimate of aerodynamic forces in prograde maneuvers, and will lead to underestimating the aerodynamic forces in retrograde orbit transfer maneuvers. The present analysis allows for bank angle, lift, drag modulation and thrust control. While the use of lift, bank angle and thrust control have been previously considered, the drag modulation is proposed here to aid in satisfying the flight time constraint. The formulation uses a sixth-order nonlinear vehicle model with four control variables. Assuming that all the state variables can be measured or accurately estimated, and that the system nonlinearities are known with reasonable accuracy, this model is transformed into a linear, time-invariant form. The guidance problem is then formulated and solved in the feedback linearized coordinates. The resulting solution is subsequently transformed back to the original coordinates to obtain the nonlinear feedback law. The nonlinear guidance law incorporates all known details about the vehicle and ambient conditions. For instance, it can utilize real-time estimates of atmospheric density and winds if these were available. Consequently the present guidance law can be expected to have an acceptable performance even while operating under offnominal conditions. Further details on feedback linearization, guidance law development, and numerical evaluation are furnished in the ensuing sections.

\section{Point-Mass Spacecraft Model}

The present analysis will only consider the atmospheric portion of the aeroassisted orbit transfer mission. Thus, the following development assumes that the thrust impulse required for entry into the planetary atmosphere has been applied. Point-mass equations of motion for a vehicle flying over an oblate spheroidal planet in a planet centered inertial frame is given by the vector differential equation

$$
\left[\begin{array}{l}
\ddot{x} \\
\ddot{y} \\
\ddot{z}
\end{array}\right]=\frac{1}{m}\left[\begin{array}{ccc}
\sin \psi \cos \theta & -\sin \theta & \cos \psi \cos \theta \\
\sin \psi \sin \theta & \cos \theta & \cos \psi \sin \theta \\
-\cos \psi & 0 & \sin \psi
\end{array}\right]\left[\begin{array}{ccc}
\sin \gamma \cos \chi & -\sin \chi & \cos \gamma \cos \chi \\
\sin \gamma \sin \chi & \cos \chi & \cos \gamma \sin \chi \\
-\cos \gamma & 0 & \sin \gamma
\end{array}\right]\left[\begin{array}{c}
-\mathrm{L} \cos \phi \\
-\mathrm{L} \sin \phi \\
\mathrm{T}-\mathrm{D}
\end{array}\right]+\left[\begin{array}{l}
\mathrm{g}_{\mathrm{x}} \\
\mathrm{g}_{\mathrm{y}} \\
\mathrm{g}_{\mathrm{z}}
\end{array}\right]
$$


$\mathrm{x}, \mathrm{y}, \mathrm{z}$ are the vehicle position components measured in the planet centered inertial frame $(X, Y, Z)$ shown in Figure 1. The $\mathrm{X}$-axis of the inertial frame points along a chosen principal direction, while the $\mathrm{Z}$-axis is aligned with the planet's rotation axis. The $\mathrm{Y}$-axis completes the right handed triad. In the case of earth, the principal direction is often chosen as the direction of the Vernal Equinox [16]. Figure 1 also shows the topocentric inertial frame $\left(\mathrm{X}_{\mathrm{T}}, \mathrm{Y}_{\mathrm{T}}, \mathrm{Z}_{\mathrm{T}}\right)$ used for the calculation of the flight path angle $\gamma$ and the heading angle $\chi$. In equation (1), the quantity $T$ is the vehicle thrust, $D$ is the vehicle drag, $L$ the lift, $m$ the vehicle mass and $\phi$ the bank angle. $\theta$ is the longitude with respect to the principal direction and $\psi$ is the latitude. Vehicle mass $\mathrm{m}$ and thrust $\mathrm{T}$ can be functions of time. $g_{x}, g_{y}, g_{z}$ are the components of acceleration due to gravity. In all that follows, a dot over variables will indicate differetiation with respect to time, and the superscript $T$ will denote the matrix transpose operation.

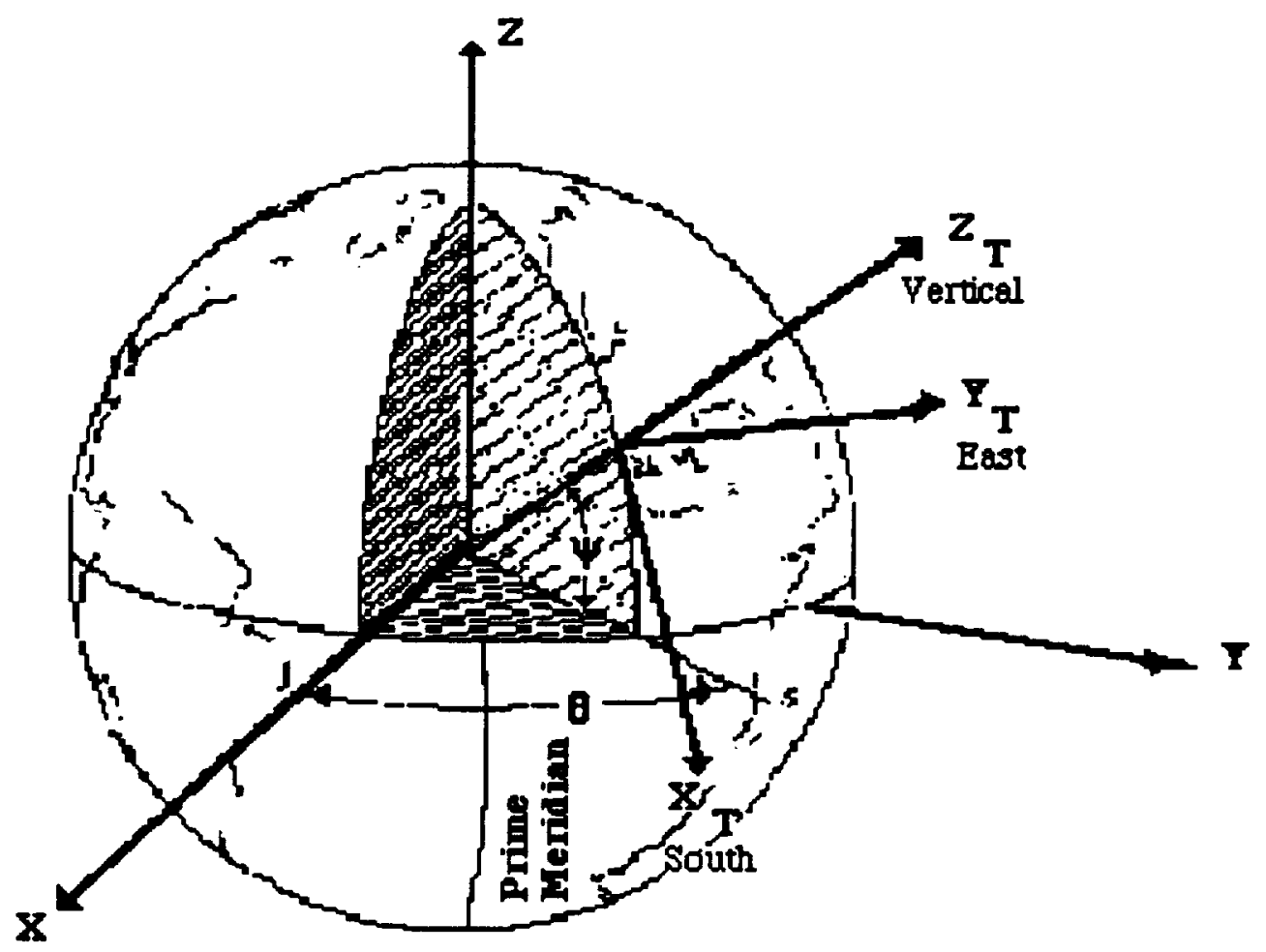

\section{Principal Direction}

\section{Fig. 1. Coordinate Systems}

Given the vehicle position in the geocentric inertial frame, the corresponding latitude and longitude can be calculated as:

$$
\theta=\tan ^{-1}\left[\frac{\mathrm{y}}{\mathrm{x}}\right], \psi=\tan ^{-1}\left[\frac{\mathrm{z}}{\sqrt{\mathrm{x}^{2}+\mathrm{y}^{2}}}\right]
$$


Longitude with respect to any specified references on the planet such as the Greenwich meridian in case of earth can be found by subtracting the instantaneous longitude of the reference meridian with respect to the Vernal Equinox direction from the longitude $\theta$. If the vehicle velocity vector with respect to the geocentric inertial frame is known, the flight path angle $\gamma$ and the heading angle $\chi$ can be computed as follows. First compute the velocity vector in the topocentric system using the transformation:

$$
\left[\begin{array}{c}
u \\
v \\
w
\end{array}\right]=\left[\begin{array}{ccc}
\sin \psi \cos \theta & \sin \psi \sin \theta & -\cos \psi \\
-\sin \theta & \cos \theta & 0 \\
\cos \psi \cos \theta & \cos \psi \sin \theta & \sin \psi
\end{array}\right]\left[\begin{array}{c}
\dot{x} \\
\dot{y} \\
\dot{z}
\end{array}\right]
$$

The $\mathrm{X}_{\mathrm{T}}$ axis of the topocentric frame points in the southern direction, the $\mathrm{Y}_{\mathrm{T}}$ axis points in the east direction and the $Z_{\mathrm{T}}$ axis points in the direction of local vertical, see Figure 1 for details. The flight path angle with respect to the local horizontal and the heading angle with respect to south can then be computed as:

$$
\chi=\tan ^{-1}\left[\frac{\mathrm{v}}{\mathrm{u}}\right], \gamma=\tan ^{-1}\left[\frac{\mathrm{w}}{\sqrt{\mathrm{u}^{2}+\mathrm{v}^{2}}}\right]
$$

If desired, the heading angle with respect to true north can be computed as $(\pi-\chi)$. Figure 2 shows the topocentric frame together with the vehicle flight path axis system.

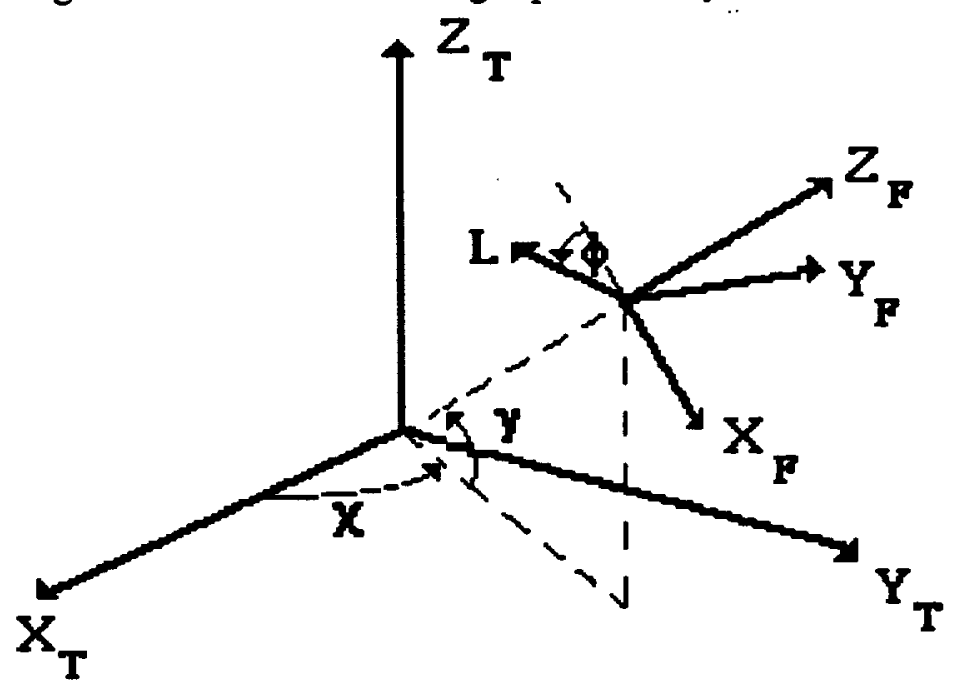

Fig. 2. Topocentric Frame and the Flight Path Axis System 
Figure 2 also shows the sign convention employed for the definition of the vehicle bank angle. The vehicle thrust and drag acts along the $\mathrm{Z}_{\mathrm{F}}$ axis, while the vertical component of lift acts along the $\mathrm{XF}$ axis. The horizontal component of lift acts along the - $\mathrm{YF}_{\mathrm{F}}$ axis. It is assumed here that the thrust acts along the velocity vector and that the vehicle employs coordinated maneuvers. In cases where these two assumptions are not admissible, an additional transformation incorporating the angle of attack and the angle of sideslip needs to be included in the analysis.

In order to compute the aerodynamic forces acting on the vehicle, the vehicle relative velocity with respect to the atmosphere needs to be found. The atmospheric rotational velocity with respect to the inertial frame and the local wind velocities can be included to yield the components of the vehicle velocity vector relative to the atmosphere as

$$
\left[\begin{array}{l}
u_{2} \\
v_{2} \\
w_{2}
\end{array}\right]=\left[\begin{array}{c}
u-u_{w} \\
v-v_{w}-r \omega \cos \psi \\
w-w_{w}
\end{array}\right]
$$

where the radial position of the vehicle with respect to the planet center is given by

$$
r=\sqrt{x^{2}+y^{2}+z^{2}}
$$

$\omega$ is the planet's rotational rate and $u_{W}, v_{W}, w_{W}$ are the ambient wind velocity components defined in the topocentric frame. These wind components can be specified as a function of latitude, longitude and altitude. The vehicle altitude at any time instant can be computed as

$$
h=\sqrt{x^{2}+y^{2}+z^{2}}-r_{c}
$$

The variable $r_{c}$ is the distance between the planet surface and the origin of the coordinate system measured along the direction of $r$. The vehicle velocity with respect to the planet atmosphere is given by

$$
v_{i}=\sqrt{u_{a}^{2}+v_{a}^{2}+w_{a}^{2}}
$$

This velocity should be used in the calculation of aerodynamic forces. The gravitational acceleration model used in the present analysis can include any number of harmonics. For instance, the gravitational model may be of the form [16]: 


$$
\begin{gathered}
g_{x}=\frac{-\mu x}{r^{3}}\left[1-J_{2} \frac{3}{2}\left(\frac{r_{e}}{r}\right)^{2}\left(5 \frac{z^{2}}{r^{2}}-1\right)+J_{3} \frac{5}{2}\left(\frac{r_{e}}{r}\right)^{3}\left(\frac{3 z}{r}-\frac{7 z^{3}}{r^{3}}\right)+\ldots \ldots . .\right] \\
g_{y}=\frac{-\mu y}{r^{3}}\left[1-J_{2} \frac{3}{2}\left(\frac{r_{e}}{r}\right)^{2}\left(5 \frac{z^{2}}{r^{2}}-1\right)+J_{3} \frac{5}{2}\left(\frac{r_{e}}{r}\right)^{3}\left(\frac{3 z}{r}-\frac{7 z^{3}}{r^{3}}\right)+\ldots \ldots . .\right] \\
g_{y}=\frac{-\mu z}{r^{3}}\left[1+J_{2} \frac{3}{2}\left(\frac{r_{e}}{r}\right)^{3}\left(3-5 \frac{z^{2}}{r^{2}}\right)+J_{3} \frac{3}{2}\left(\frac{r_{e}}{r}\right)^{2}\left(\frac{10 z}{r}-\frac{35 z^{3}}{3 r^{3}}-\frac{r}{z}\right)+\ldots \ldots . . .\right.
\end{gathered}
$$

Here $\mu$ is the gravitational constant for the planet, $\mathrm{r}$ is the radius vector, $\mathrm{r}_{\mathrm{e}}$ the planet radius, and $\mathrm{J}_{2}, \mathrm{~J}_{3} \ldots$ are the harmonics of the planet's gravitational potential.

The vehicle aerodynamic lift and drag are given by the expressions

$$
\mathrm{L}=\frac{1}{2} \rho \mathrm{V}_{\mathrm{a}}^{2} \mathrm{~s} \mathrm{C}_{\mathrm{L}}, \mathrm{D}=\frac{1}{2} \rho \mathrm{V}_{\mathrm{a}}^{2} \mathrm{~s}\left(\mathrm{C}_{\mathrm{D}}+\mathrm{C}_{\mathrm{Dm}}\right)
$$

The drag coefficient $\mathrm{CD}$ could be a function of the lift coefficient, Mach number and altitude. The control variable $\mathrm{CDm}$ is the component of drag that can be modulated using aerodynamic devices. $\mathrm{C}_{\mathrm{L}}$ is the lift coefficient, another control variable in the problem. The bank angle $\phi$ and the vehicle thrust $T$ are the additional control variables in the problem. The air density $\rho$ can be a function of altitude, latitude and longitude. The variable $s$ is the reference area used in the calculation of aerodynamic forces.

The vehicle model elaborated in the foregoing is nonlinear and time varying. In the present form it is useful only for numerical studies. In the next section it will be shown that the model can be made amenable to analysis through a series of transformations.

\section{Eeedback_Linearization}

The spacecraft nonlinear point-mass model may be transformed to a more convenient form by assuming that all the state variables required for the calculation of the right hand side of equation (1) are available from measurements. In this case, the right-hand-side can be treated as a three component pseudo-control vector, yielding the equations of motion as:

$$
\left[\begin{array}{l}
\ddot{x} \\
\ddot{y} \\
\ddot{z}
\end{array}\right]=\left[\begin{array}{l}
U_{1} \\
U_{2} \\
U_{3}
\end{array}\right]
$$


$\mathrm{U}_{1}, \mathrm{U}_{2}, \mathrm{U}_{3}$ are the pseudo-control variables. These quantities are unknown at this stage because although the state variables and the system nonlinearities are known, the control variables are yet to be determined. Physically, these quantities are the spacecraft acceleration components in the geocentric inertial frame. If the pseudo control components $\mathrm{U}_{1}, \mathrm{U}_{2}, \mathrm{U}_{3}$ were available, the actual control variables $\mathrm{T}, \mathrm{CL}, \mathrm{CDm}, \phi$ can be computed using an inverse transformation as follows. First compute the vehicle flight path angle, heading angle, latitude and longitude using the measured vehicle velocity and position components. The force vector $\left[F_{x}, F_{y}, F_{z}\right]^{\top}$ in the flight path axis system is next computed using the expression:

$$
\left[\begin{array}{l}
\mathrm{F}_{\mathrm{x}} \\
\mathrm{F}_{\mathrm{y}} \\
\mathrm{F}_{\mathrm{z}}
\end{array}\right]=\mathrm{m}\left[\begin{array}{ccc}
\sin \gamma \cos \chi & \sin \gamma \sin \chi & -\cos \gamma \\
-\sin \chi & \cos \chi & 0 \\
\cos \gamma \cos \chi & \cos \gamma \sin \chi & \sin \gamma
\end{array}\right]\left[\begin{array}{ccc}
\sin \psi \cos \theta & \sin \psi \sin \theta & -\cos \psi \\
-\sin \theta & \cos \theta & 0 \\
\cos \psi \cos \theta & \cos \psi \sin \theta & \sin \psi
\end{array}\right]\left[\begin{array}{c}
\mathrm{U}_{1}-\mathrm{g}_{\mathrm{x}} \\
\mathrm{U}_{2}-\mathrm{g}_{\mathrm{y}} \\
\mathrm{U}_{3}-\mathrm{g}_{\mathrm{z}}
\end{array}\right]
$$

The vehicle lift coefficient can then be calculated as :

$$
C_{L}=\frac{2 \sqrt{F_{x}^{2}+F_{y}^{2}}}{\rho V_{a}^{2} s}
$$

The bank angle $\phi$ is given by

$$
\phi=\tan ^{-1}\left[F_{y} / F_{z}\right]
$$

Next, the thrust and drag modulation coefficient can be computed based on the sign and magnitude of the force component $F_{Z}$ as follows:

$$
\text { If } \begin{array}{rl}
\mathrm{F}_{\mathrm{z}}<\frac{-1}{2} \rho \mathrm{V}_{a}^{2} \mathrm{sC}_{\mathrm{D}}, \quad \mathrm{T} & =0 \\
& \\
\text { If } \mathrm{C}_{\mathrm{Dm}} \geq \frac{1}{2} \rho \mathrm{V}_{\mathrm{a}}^{2} \mathrm{sC}_{\mathrm{D}}, \quad \frac{-2 \mathrm{~F}_{\mathrm{z}}}{\rho \mathrm{V}_{a}^{2} \mathrm{~s}}-\mathrm{C}_{\mathrm{D}} & \mathrm{T}=\mathrm{F}_{\mathrm{z}}-\frac{1}{2} \rho \mathrm{V}_{a}^{2} \mathrm{sC}_{\mathrm{D}} \\
\mathrm{C}_{\mathrm{Dm}} & =0
\end{array}
$$

Thus, if the pseudo-control variables $\mathrm{U}_{1}, \mathrm{U}_{2}, \mathrm{U}_{3}$ are known, the real control variables $\mathrm{T}, \mathrm{C}_{\mathrm{L}}$, $\mathrm{CDm}$, and $\phi$ can be recovered using the inverse transformation (14) - (18). 
In the case of pure aerobraking maneuvers as in Reference $1,2,4-12$, vehicle thrust and drag modulation capabilities may not available. In this case, the inverse transformation has to account for this fact by considering the third component of the vector equation (14) as a constraint of the form :

$$
\left[\begin{array}{c}
\cos \gamma \cos \chi \sin \psi \cos \theta-\cos \gamma \sin \chi \sin \theta+\sin \gamma \cos \psi \cos \theta \\
\cos \gamma \cos \chi \sin \psi \sin \theta+\cos \gamma \sin \chi \cos \theta+\sin \gamma \cos \psi \sin \theta \\
-\cos \gamma \cos \chi \cos \psi+\sin \gamma \sin \psi
\end{array}\right]^{\mathrm{T}}\left[\begin{array}{c}
\mathrm{U}_{1}-\mathrm{g}_{\mathrm{x}} \\
\mathrm{U}_{2}-\mathrm{g}_{\mathrm{y}} \\
\mathrm{U}_{3}-\mathrm{g}_{\mathrm{z}}
\end{array}\right]+\frac{\mathrm{D}}{\mathrm{m}}=0
$$

This constraint can be used to determine one of the pseudo control variables in terms of the other two. The vehicle lift coefficient and bank angle can then be calculated as indicated. However, the guidance objectives have to be modified in this case because the simultaneous control of flight time, position and velocity control at the atmospheric exit will no longer be possible. Such a formulation will not be examined in the present research. In the ensuing, it will be assumed that all the four control variables are available.

\section{Guidance Law Development}

The previous section dealt with an approach for making the nonlinear vehicle model amenable to analysis. In this section, the guidance problem will be formulated in the transformed coordinates and solved. Inverse transformation of this solution to the original coordinates will then yield the nonlinear feedback guidance law. The following development will assume that all the state variables required for computing the feedback law are known or can be estimated. Once the guidance problem is solved with perfect information, the effects of incomplete or imperfect information can be investigated using this solution.

It is assumed here that altitude, flight path angle, heading angle, latitude, longitude at atmospheric exit and the time of flight through the atmosphere are all specified. Since the present formulation uses a planet centered inertial frame, these conditions can first be translated to the Cartesian frame using well known relationships in astrodynamics [16]. For instance, given the flight path angle $\gamma_{\mathrm{f}}$, heading angle $\chi_{\mathrm{f}}$, latitude $\psi_{\mathrm{f}}$, longitude $\theta_{\mathrm{f}}$ and the velocity at atmospheric exit $V_{\mathrm{f}}$, the components of the terminal velocity vector in the planet centered inertial frame can be calculated using the expression

$$
\left[\begin{array}{c}
\dot{x}_{f} \\
\dot{y}_{f} \\
\dot{z}_{f}
\end{array}\right]=\left[\begin{array}{ccc}
\sin \psi_{f} \cos \theta_{f} & -\sin \theta_{f} & \cos \psi_{f} \cos \theta_{f} \\
\sin \psi_{f} \sin \theta_{f} & \cos \theta_{f} & \cos \psi_{f} \sin \theta_{f} \\
-\cos \psi_{f} & 0 & \sin \psi_{f}
\end{array}\right]\left[\begin{array}{ccc}
\sin \gamma_{f} \cos \chi_{f} & -\sin \chi_{f} & \cos \gamma_{f} \cos \chi_{f} \\
\sin \gamma_{f} \sin \chi_{f} & \cos \chi_{f} & \cos \gamma_{f} \sin \chi_{f} \\
-\cos \gamma_{f} & 0 & \sin \gamma_{f}
\end{array}\right]\left[\begin{array}{c}
V_{f} \\
0 \\
0
\end{array}\right]
$$


The objective of the present research is to synthesize an implementable guidance law that achieves a specified set of final conditions. As mentioned elsewhere in this paper, the performance index is assumed to the integral of the square of the vehicle acceleration. Primary objective is to synthesize a maneuver guidance law that results in least structural load on the vehicle. Since the magnitude of a vector is invariant with respect to rotational transformations, the vehicle acceleration magnitude is the same as the magnitude of the pseudo control variables. In addition to being mathematically convenient, this performance index may also lead to lower energy loss during the maneuver. With this, the aeroassisted maneuver guidance problem may be defined as: determine the vehicle lift, bank angle, drag modulation coefficient and thrust that achieve

$$
\min _{U_{1}, U_{2}, U_{3}}\left\{\int_{0}^{t_{t}}\left[U_{1}^{2}+U_{2}^{2}+U_{3}^{2}\right] d t\right\}
$$

given the position vector and velocity vectors at the atmospheric entry and exit points, subject to the differential constraint (13). The final time tf is assumed to be specified. If desired, flight time can be included in the performance index as in Reference 15.

In order to solve this optimal guidance problem, the variational Hamiltonian [17] is first defined as:

$$
\mathrm{H}=\frac{\mathrm{a}}{2}\left[\mathrm{U}_{1}^{2}+\mathrm{U}_{2}^{2}+\mathrm{U}_{3}^{2}\right]+\lambda_{1} \dot{\mathrm{x}}+\lambda_{2} \dot{\mathrm{y}}+\lambda_{3} \dot{\mathrm{z}}+\lambda_{4} \mathrm{U}_{1}+\lambda_{5} \mathrm{U}_{2}+\lambda_{6} \mathrm{U}_{3}
$$

The costate equations and the optimality conditions for this problem can be obtained as

$$
\begin{gathered}
\dot{\lambda}_{1}=\dot{\lambda}_{2}=\dot{\lambda}_{3}=0 \\
\dot{\lambda}_{4}=-\lambda_{1}, \dot{\lambda}_{5}=-\lambda_{2}, \dot{\lambda}_{6}=-\lambda_{3} \\
\mathrm{U}_{1}=-\lambda_{4}, \mathrm{U}_{2}=-\lambda_{5}, \mathrm{U}_{3}=-\lambda_{6}
\end{gathered}
$$

The initial and terminal conditions on the costates are unknown. Expressions (24) can be integrated together with (23) to yield

$$
\lambda_{4}=c_{1}-\lambda_{1} \mathrm{t}, \lambda_{5}=c_{2}-\lambda_{2} \mathrm{t}, \lambda_{6}=c_{3}-\lambda_{3} \mathrm{t}
$$

$c_{1}, c_{2}, c_{3}$ are constants of integration. Substituting in (25), the control variables become

$$
U_{1}=\lambda_{1} t-c_{1}, U_{2}=\lambda_{2} t-c_{2}, U_{3}=\lambda_{3} t-c_{3}
$$


Substituting (27) in the feedback linearized equations of motion and integrating results in:

$$
\begin{gathered}
\dot{x}=\dot{x}_{0}+t\left[\lambda_{1} \frac{t}{2}-c_{1}\right], \dot{y}=\dot{y}_{0}+t\left[\lambda_{2} \frac{t}{2}-c_{2}\right], \dot{z}=\dot{z}_{0}+t\left[\lambda_{3} \frac{t}{2}-c_{3}\right] \\
x=x_{0}+\dot{x}_{0} t+\frac{t^{2}}{2}\left[\lambda_{1} \frac{t}{3}-c_{1}\right], y=y_{0}+\dot{y}_{0} t+\frac{t^{2}}{2}\left[\lambda_{2} \frac{t}{3}-c_{2}\right], z=z_{0}+\dot{z}_{0} t+\frac{t^{2}}{2}\left[\lambda_{3} \frac{t}{3}-c_{3}\right]
\end{gathered}
$$

The constants $c_{1}, c_{2}, c_{3}, \lambda_{1}, \lambda_{2}, \lambda_{3}$ are the unknowns in these equations. These may be calculated from the given terminal conditions. Using the specified exit velocity components and the final time, the costates $\lambda_{1}, \lambda_{2}, \lambda_{3}$ can be computed as

$$
\lambda_{1}=\frac{2}{t_{f}^{2}}\left[\dot{x}_{f}-\dot{x}_{0}+c_{1} t_{f}\right], \lambda_{2}=\frac{2}{t_{f}^{2}}\left[\dot{y}_{f}-\dot{y}_{0}+c_{2} t_{f}\right], \lambda_{3}=\frac{2}{t_{f}^{2}}\left[\dot{z}_{f}-\dot{z}_{0}+c_{3} t_{f}\right]
$$

These may next be substituted in the equations (29), together with the specified terminal conditions yield

$$
c_{1}=\frac{-6}{t_{f}^{2}}\left(x_{f}-x_{0}\right)+\frac{2}{t_{f}}\left(2 \dot{x}_{0}+\dot{x}_{f}\right), c_{2}=\frac{-6}{t_{f}^{2}}\left(y_{f}-y_{0}\right)+\frac{2}{t_{f}}\left(2 \dot{y}_{0}+\dot{y}_{f}\right), c_{3}=\frac{-6}{t_{f}^{2}}\left(z_{f}-z_{0}\right)+\frac{2}{t_{f}}\left(2 \dot{z}_{0}+\dot{z}_{f}\right)
$$

At this stage, an open-loop maneuver guidance law can be obtained by substituting for $\mathrm{c}_{1}, \mathrm{c}_{2}, \mathrm{c} 3$, $\lambda_{1}, \lambda_{2}, \lambda_{3}$ in (27). It can be verified that the guidance law satisfies the strengthened LegendreClebsch and Jacobi necessary conditions [17]. As a result, all the trajectories emerging from the present analysis afford a strong local minimum. Further, since there exists a unique optimal trajectory for every specified set of boundary conditions, these trajectories provide a global minimum [18].

The guidance law can next be converted to feedback form by assuming that the current time is the initial time. In this case, the difference between the current time and final time is the time-to-go parameter, denoted by the variable tgo. The resulting guidance law in terms of pseudo-control variables may be substituted in the inverse transformation to yield the nonlinear guidance law for aeroassisted orbit transfer as: 


$$
\begin{aligned}
{\left[\begin{array}{c}
-\mathrm{L} \cos \phi \\
-\mathrm{L} \sin \phi \\
\mathrm{T}-\mathrm{D}
\end{array}\right]=} & {\left[\begin{array}{ccc}
\sin \gamma \cos \chi & \sin \gamma \sin \chi & -\cos \gamma \\
-\sin \chi & \cos \chi & 0 \\
\cos \gamma \cos \chi & \cos \gamma \sin \chi & \sin \gamma
\end{array}\right] } \\
& {\left[\begin{array}{ccc}
\sin \psi \cos \theta & \sin \psi \sin \theta & -\cos \psi \\
-\sin \theta & \cos \theta & 0 \\
\cos \psi \cos \theta & \cos \psi \sin \theta & \sin \psi
\end{array}\right]\left[\begin{array}{l}
\mathrm{G}_{1}\left(\mathrm{x}_{\mathrm{f}}-\mathrm{x}\right)-\mathrm{G}_{2}\left(2 \dot{\mathrm{x}}+\dot{\mathrm{x}}_{\mathrm{f}}\right) \\
\mathrm{G}_{1}\left(\mathrm{y}_{\mathrm{f}}-\mathrm{y}\right)-\mathrm{G}_{2}\left(2 \dot{\mathrm{y}}+\dot{\mathrm{y}}_{\mathrm{f}}\right) \\
\mathrm{G}_{1}\left(\mathrm{z}_{\mathrm{f}}-\mathrm{z}\right)-\mathrm{G}_{2}\left(2 \dot{\mathrm{z}}+\dot{\mathrm{z}}_{\mathrm{f}}\right)
\end{array}\right] }
\end{aligned}
$$

where

$$
G_{1}=\frac{6}{t_{80}^{2}}, G_{2}=\frac{2}{t_{80}}
$$

The vehicle lift coefficient, bank angle, drag modulation coefficient and thrust can be calculated from the left hand side of this equation as in (15)-(18). Note that all the quantities on the right hand side can be computed from the current and the specified final vehicle states, and time-to-go. The performance of this guidance law will be illustrated using a point-mass simulation of a aeroassisted orbit transfer vehicle in the next section.

\section{Results and Discussions}

The guidance law developed in the previous section is next implemented on a point-mass model of a hypothetical maneuverable re-entry vehicle shown in Figure 3. The performance data for this vehicle [6] is given in Table 1. As presently configured, this vehicle has no drag modulation capabilities. However, for the purposes of guidance law evaluation it will be assumed that such a capability exists. Additionally, it is assumed that the orbit transfer maneuvers occur with earth as the primary. 


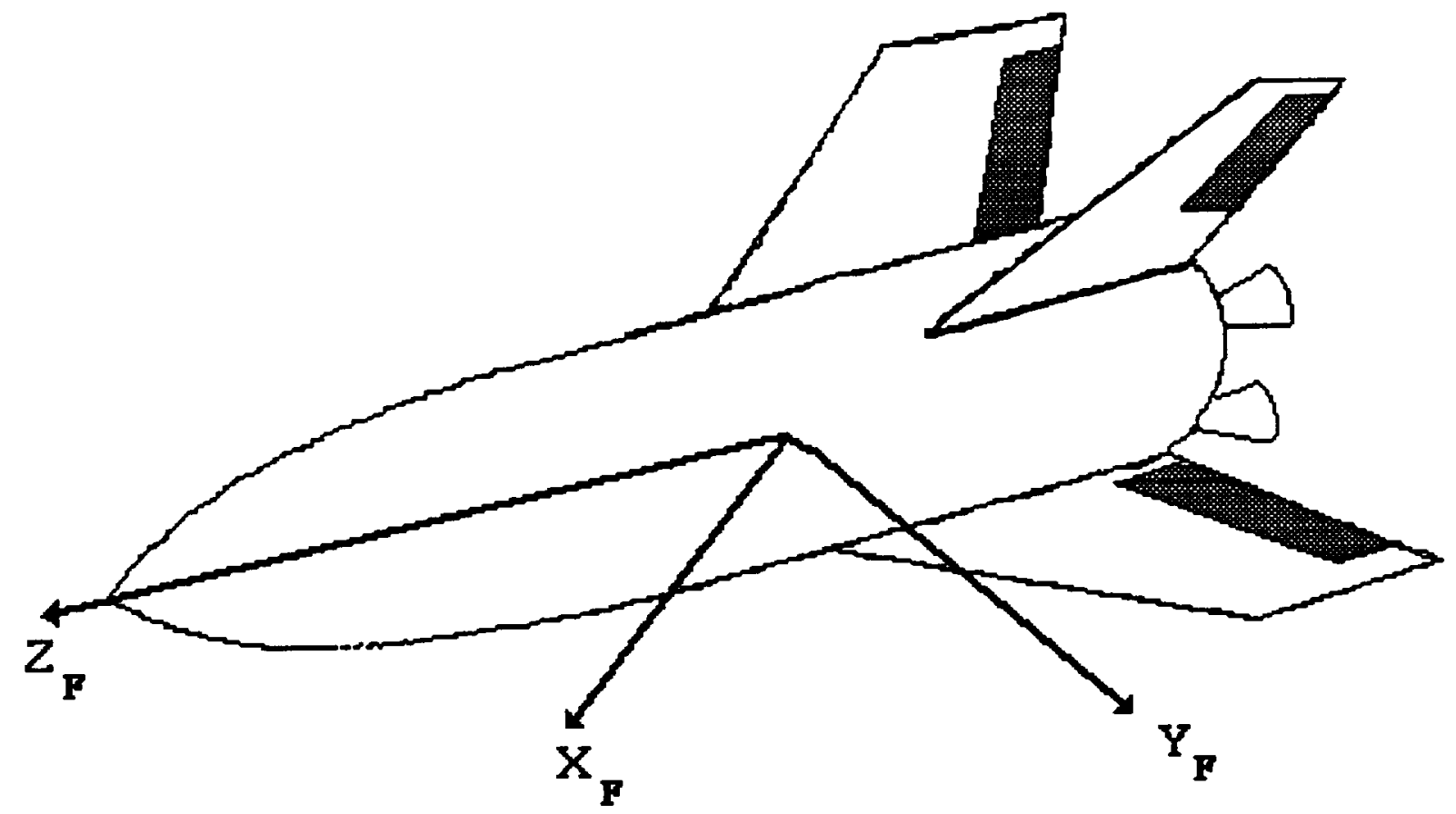

Fig. 3. Maneuverable Re-entry Research Vehicle

The vehicle simulation featured a fourth order Runge-Kutta integration scheme with a choice between linear or exponential interpolation of the U. S. standard atmosphere up to a maximum altitude of 300000 feet. Four gravitational harmonics were included in the simulation.

$\begin{array}{cc}\text { Vehicle Mass } & 335.67 \text { Slugs } \\ \text { Reference Area } & 125.84 \mathrm{ft}^{2} \\ \text { Zero-Lift Drag } & 0.032 \\ \text { Coefficient } & \\ \text { Induced Drag } & 1.4 \\ \text { Coefficient } & \\ \text { Maximum Lift } & 0.151 \\ \text { Coefficient } & \end{array}$

\section{Table 1. Maneuverable Re-entry Vehicle Data}

Three sets of boundary conditions are considered in this paper. The First one is that of a pure orbital plane change maneuver, the second one a simultaneous change in both in-plane and out-ofplane parameters, and the last one being an in-plane maneuver. Flight duration for all the three cases were assumed to be 400 seconds. The trajectory boundary conditions used in the study are summarized in Table 2. 


\section{CASE 1}

\begin{tabular}{|c|c|c|c|c|c|c|}
\hline & $\frac{\text { Latifude. }}{\text { deg. }}$ & $\frac{\text { Longitude }}{\text { deg. }}$ & $\begin{array}{c}\text { Altitude } \\
\text { feet }\end{array}$ & $\begin{array}{c}\text { Elight Path } \\
\text { Angle. } \\
\text { deg. }\end{array}$ & $\begin{array}{l}\text { Heading } \\
\text { Angle. } \\
\text { deg. }\end{array}$ & $\frac{\text { Velocity }}{\text { feet/s }}$ \\
\hline ITLA & 0 & 0 & 200000 & -0.148 & 90 & 25946 \\
\hline EINAL & 10 & 20 & 200000 & 0.0 & 130 & 14000 \\
\hline
\end{tabular}

CASE 2

$\begin{array}{cccccc}\text { Latitude } & \begin{array}{c}\text { Longitude } \\ \text { deg. }\end{array} & \begin{array}{c}\text { Altitude } \\ \text { feg. }\end{array} & \begin{array}{c}\text { Flight Path } \\ \text { Angle. }\end{array} & \begin{array}{c}\text { Heading } \\ \text { Angle }\end{array} & \begin{array}{c}\text { Velocity } \\ \text { feet/s }\end{array} \\ 0 & 0 & 200000 & -0.148 & 90 & 25946 \\ 10 & 20 & 200000 & 10 & 130 & 14000\end{array}$

\section{CASE 3}

$\begin{array}{cccccc}\begin{array}{c}\text { Latitude } \\ \text { deg }\end{array} & \begin{array}{c}\text { Longitude } \\ \text { deg }\end{array} & \begin{array}{c}\text { Altitude } \\ \text { feet }\end{array} & \begin{array}{c}\text { Flight Path } \\ \text { Angle } \\ \text { deg }\end{array} & \begin{array}{c}\text { Heading } \\ \text { Angle } \\ \text { deg }\end{array} & \begin{array}{c}\text { Velocity } \\ \text { feet/s }\end{array} \\ 0 & 0 & 200000 & -0.148 & 90 & 25946 \\ 0 & 20 & 200000 & 10 & 90 & 14000\end{array}$

\section{Table 2. Trajectory Boundary Conditions}

In the first orbit transfer maneuver scenario, the vehicle enters the atmosphere over the equator heading due east. The vehicle travels north and exits the atmosphere at 10 degree north latitude and 20 degrees north longitude. The desired exit flight path angle is zero. The only difference between the first and second case is the exit flight path angle. A rather high terminal flight path angle in Case 2 corresponds to a highly eccentric target orbit. In Case 3, the exit flight path angle is same is Case 2. However, the terminal heading angle and latitude are different.

During the course of these simulations it was found that the linear interpolation of the atmosphere produced corners in the control history. For this reason, all the results given in this paper have been generated using an exponential atmosphere model. Figure 4 illustrates the vehicle altitude history for the first set of boundary conditions. The vehicle initially descends to an altitude below 175000 feet before climbing back to meet the terminal altitude specification. The vehicle latitudelongitude evolution is given in Figure 5. The guidance law exactly meets the specified terminal altitude, longitude and latitude. 


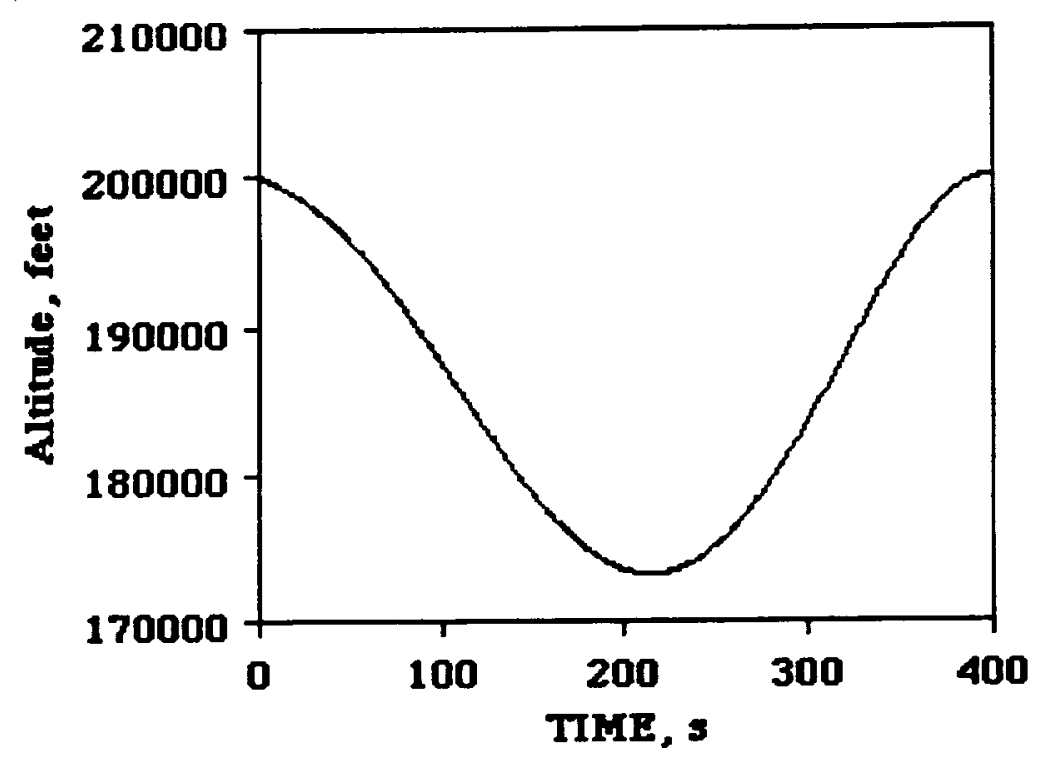

Fig. 4. Altitude Vs Time (Case 1)

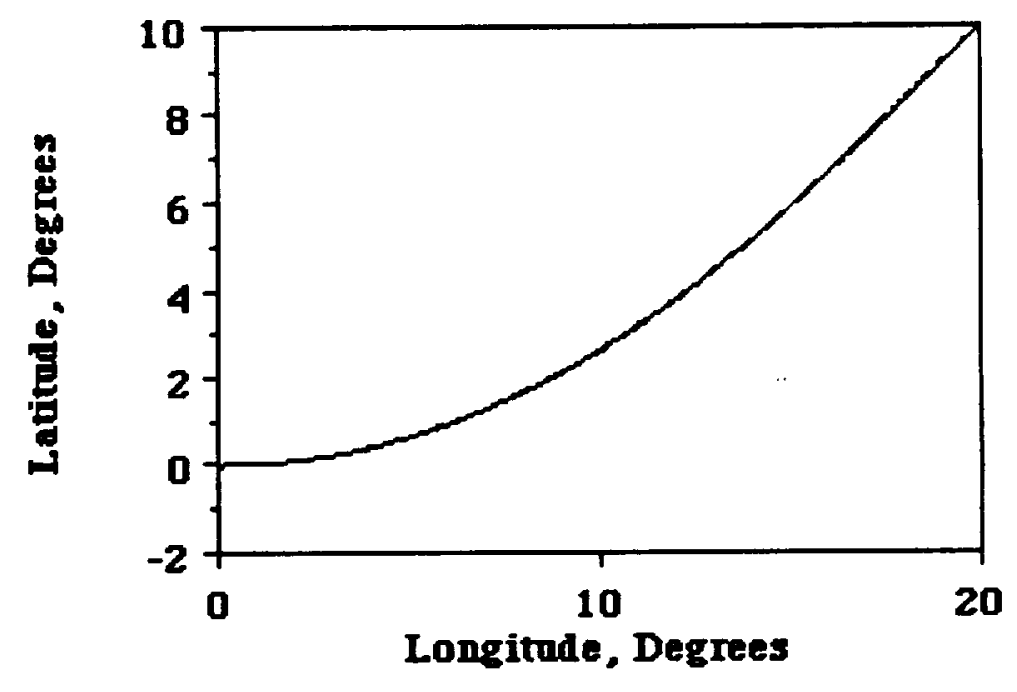

Fig. 5. Longitude Vs Latitude (Case 1)

The temporal evolution of the vehicle inertial velocity is shown in Figure 6. The corresponding flight path angle and heading angle are shown in Figures 7 and 8 . Note that the heading angle with respect to North is shown in Figure 8. The heading angle history is not monotonic because the vehicle is required to simultaneously meet all the components of the terminal velocity and position vectors. 


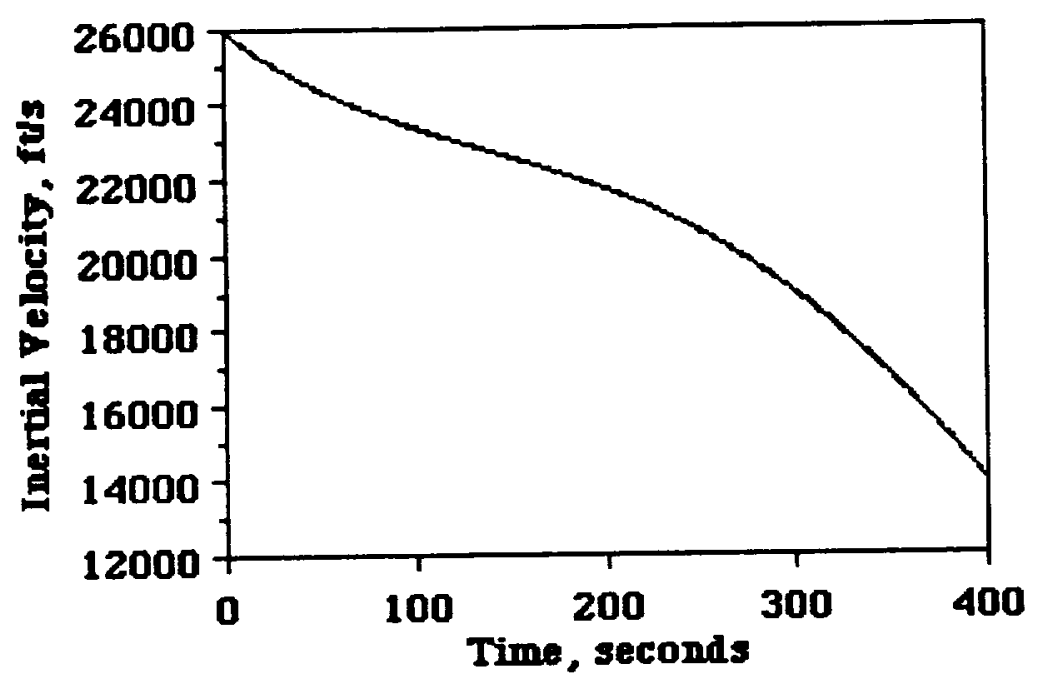

Fig. 6. Vehicle Inertial Velocity Vs Time (Case 1)

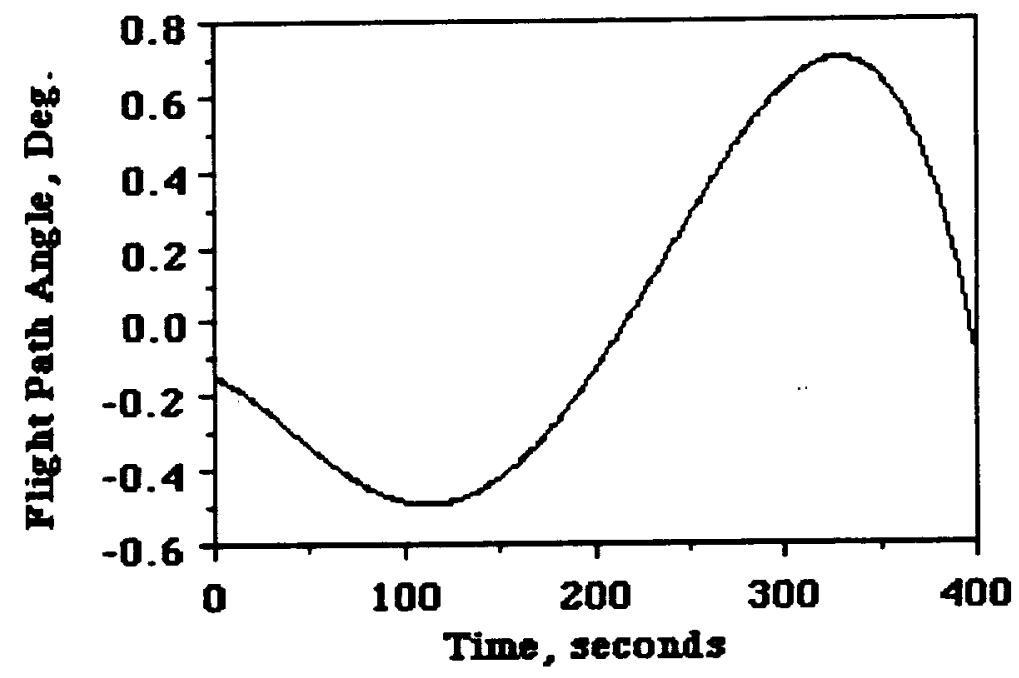

Fig. 7. Flight Path Angle Vs Time (Case 1) 


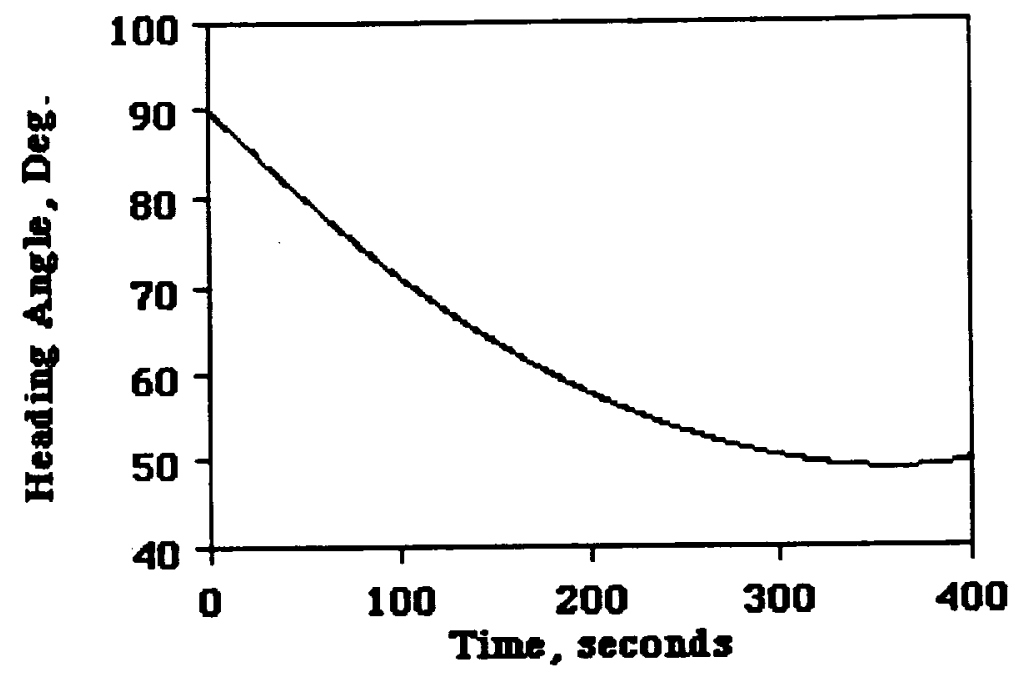

\section{Fig. 8. Heading Angle Vs Time (Case 1) (Measured Fith Respect to North)}

The lift coefficient corresponding to this trajectory is shown in Figure 9. From this figure it can be observed that the vehicle tends to use a higher lift coefficient at higher altitudes. This strategy is consistent with the specified objective of minimizing the vehicle acceleration magnitude since larger lift coefficients at lower altitudes tends to not only increase lift, but also contributes to drag.

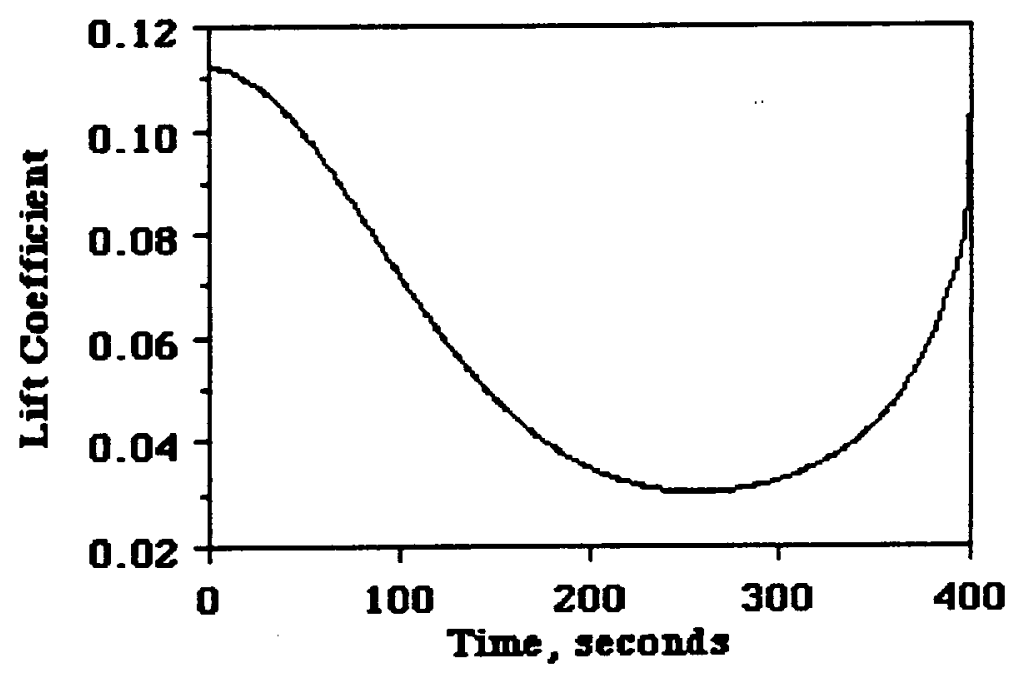

Fig. 9. Lift Coefficient Vs Time (Case 1)

The vehicle bank angle history is shown in Figure 10. As the vehicle begins the atmospheric maneuver, it banks more than 90 degrees to the left and decreases the bank angle to zero before beginning a bank to the right. If desired, the positive bank angle component can be eliminated by an appropriate re-definition of the terminal latitude and longitude. 


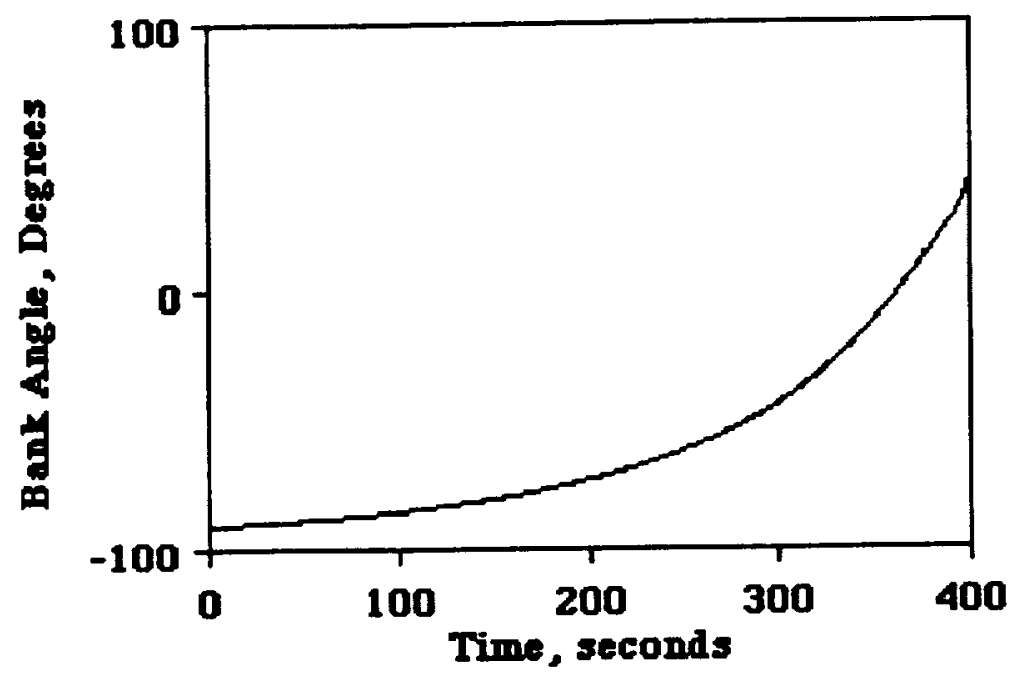

Fig. 10. Bank Angle Vs Time (Case 1)

The vehicle drag modulation coefficient along the trajectory is illustrated in Figure 11. The vehicle does not call for any drag increase during descent, but requires it as the vehicle is climbing out to meet the specified terminal velocity and position conditions.

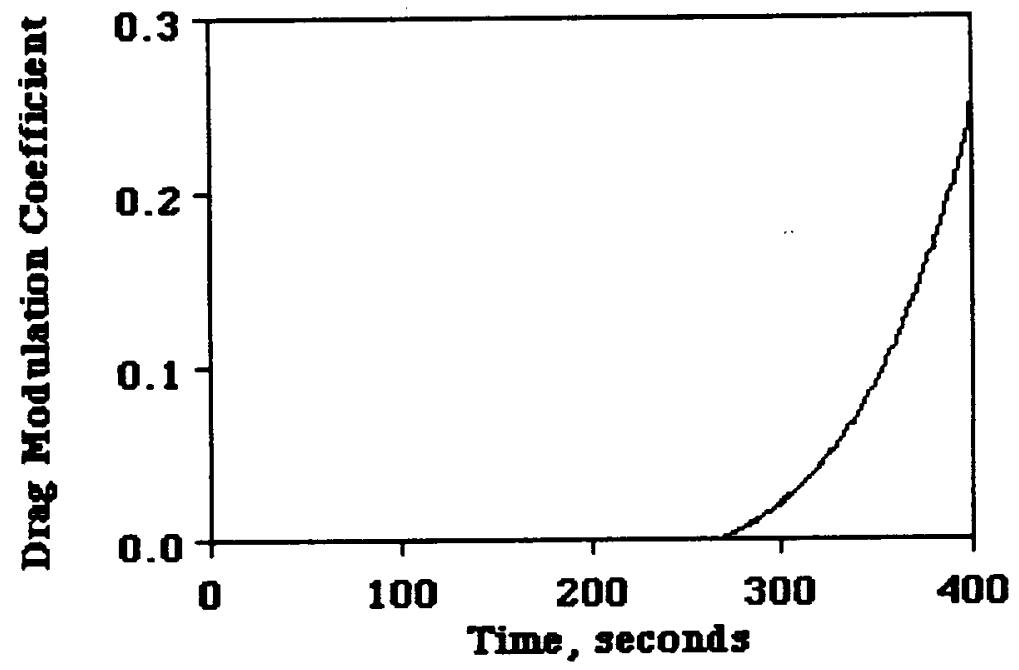

\section{Fig. 11. Drag Modulation Coefficient Vs Time (Case 1)}

The corresponding thrust history is illustrated in Figure 12. The thrust is used for velocity control during the vehicle descent. It is important to recall here that the present study achieves the control of all the six orbital parameters. As a result, the thrust and drag modulation histories given in Figure 11 and 12 depend on the specified boundary conditions. 


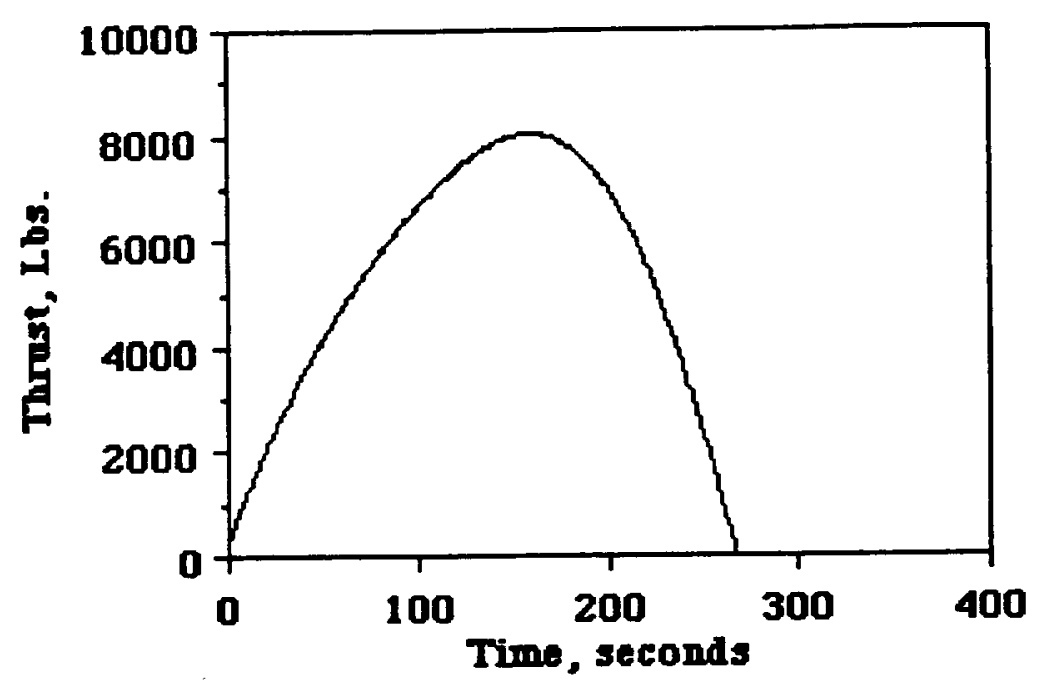

Fig. 12. Vehicle Thrust Vs Time (Case 1)

The vehicle Mach number history along the orbit transfer maneuver trajectory is illustrated in Figure 13. The vehicle starts the maneuver at about Mach 26 and has a nearly 14 Mach at atmospheric exit.

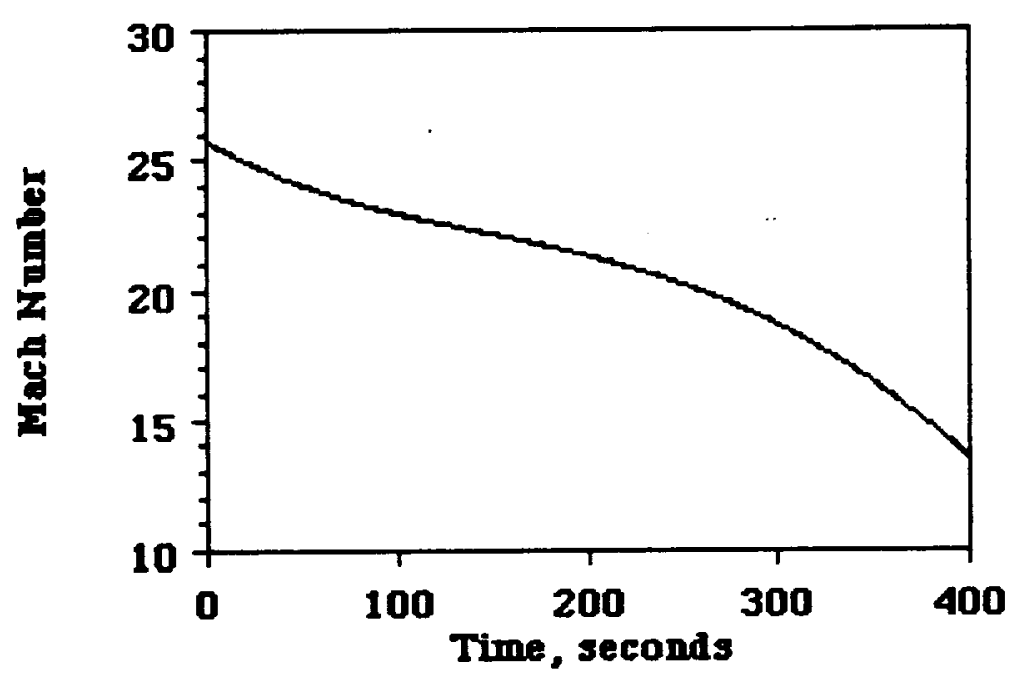

Fig. 13. Mach Number Vs Time (Case 1)

In Figures 14 through 21, compares the trajectories emerging from the three sets of boundary conditions given in Table 2. The altitude histories for the three cases are compared in Figure 14. Lowest altitude corresponds to the case of simultaneous change in all the orbital elements. Due to the high terminal flight path angle requirement, Case 3 also requires significant descent into the atmosphere. 


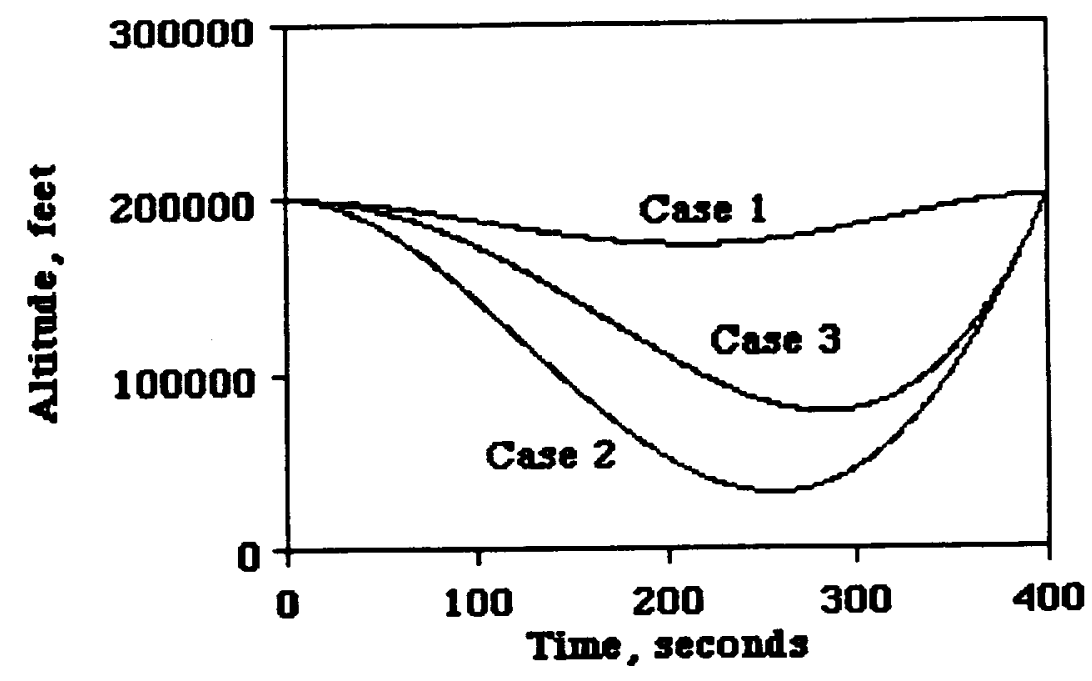

Fig. 14. A Comparison of Altitude Histories

The vehicle inertial velocities are compared in Figure 15. The velocity histories are nearly identical for case 1 and 2, while the maneuver corresponding to case 3 evolves at a much lower average velocity. The Mach number histories given in Figure 16 reveal that although the inertial velocities for case 1 and 2 were nearly identical, the vehicle experiences different airspeeds and densities due to the differences in the altitude histories.

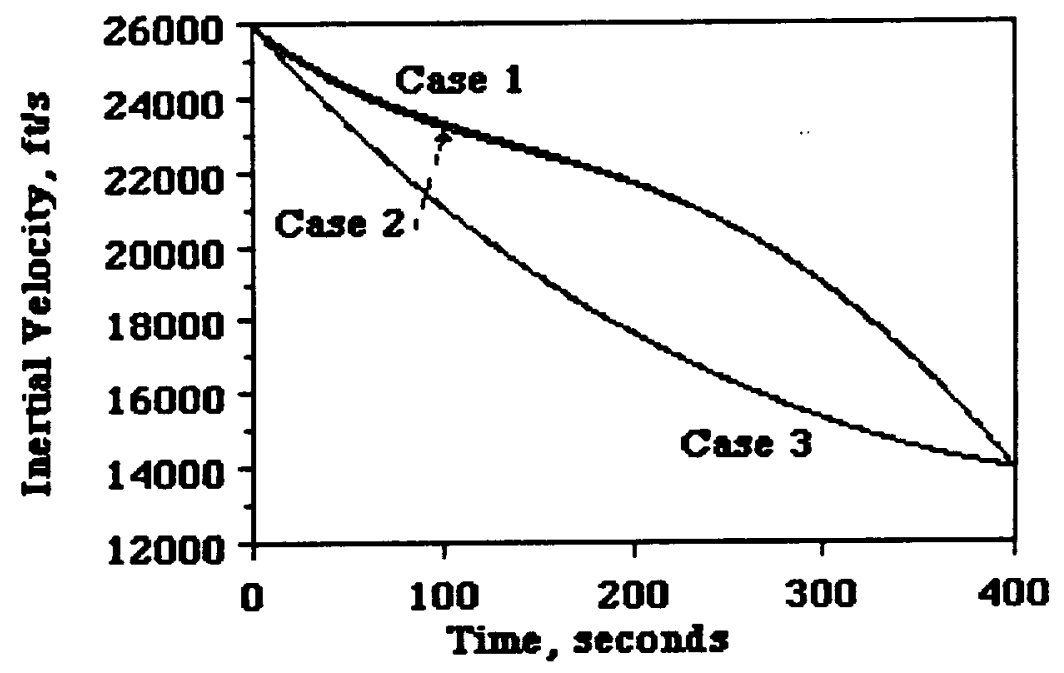

Fig. 15. A Comparison of Inertial Velocities 


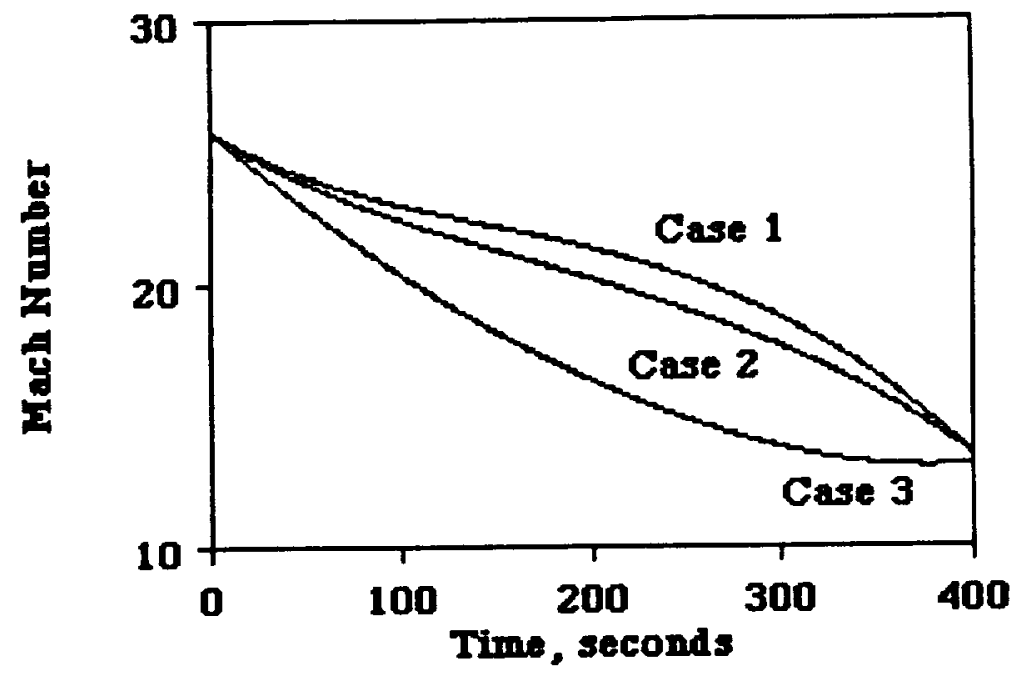

Fig. 16. A Comparison Mach Number Histories

The flight path angle along the three maneuvers are shown in Figure 17. The vehicle lift coefficient histories given in Figure 18 reveal that a much lower lift magnitude is used for in-plane changes when compared with out-of-plane changes.

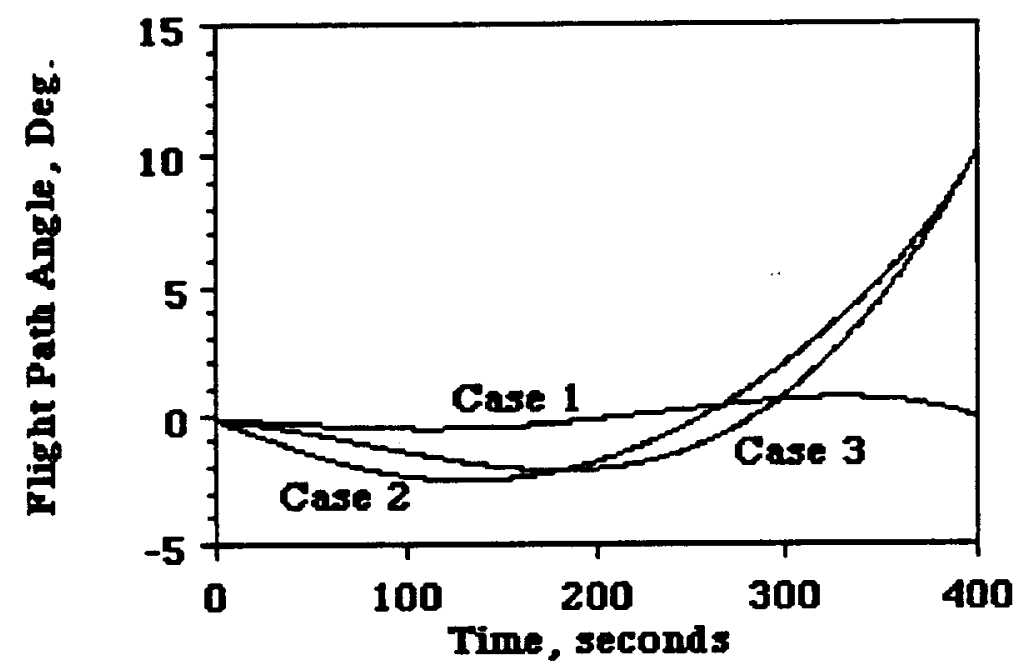

Fig. 17. A Comparison of Vehicle Flight Path Angles 


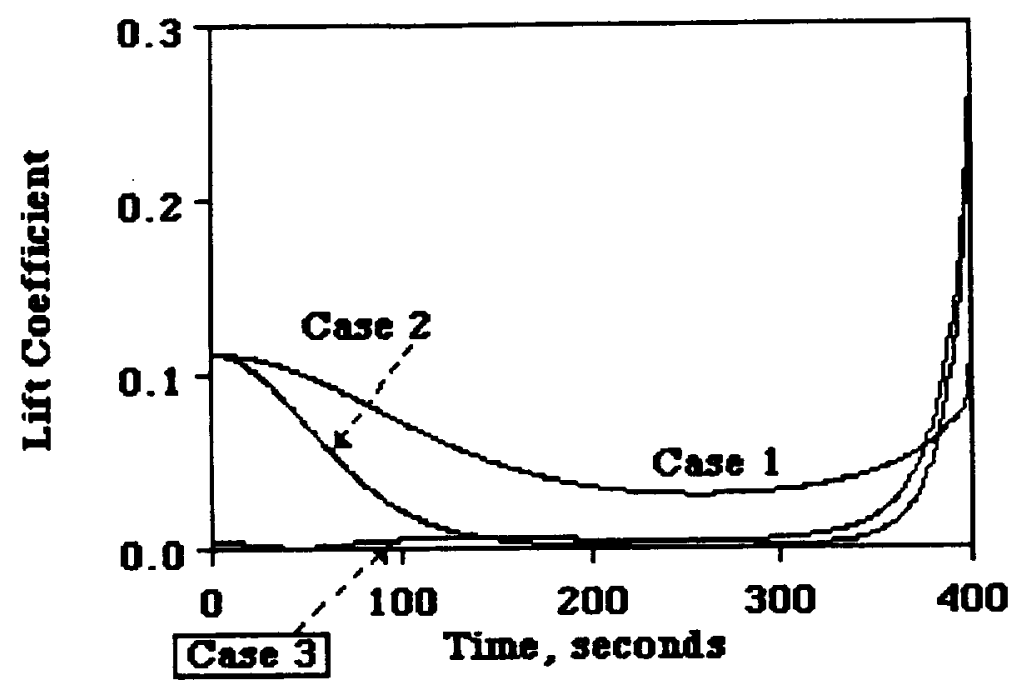

\section{Fig. 18. A Comparison of Lift Coefficient Histories}

The corresponding bank angle histories are given in Figure 19. Of the three histories given in Figure 19, the one corresponding to case 3 is the most interesting one. Upon atmospheric entry, the vehicle initially assumes an inverted position which lasts for about 40 seconds. Subsequently it uses zero bank angle during the remaining duration of the maneuver.

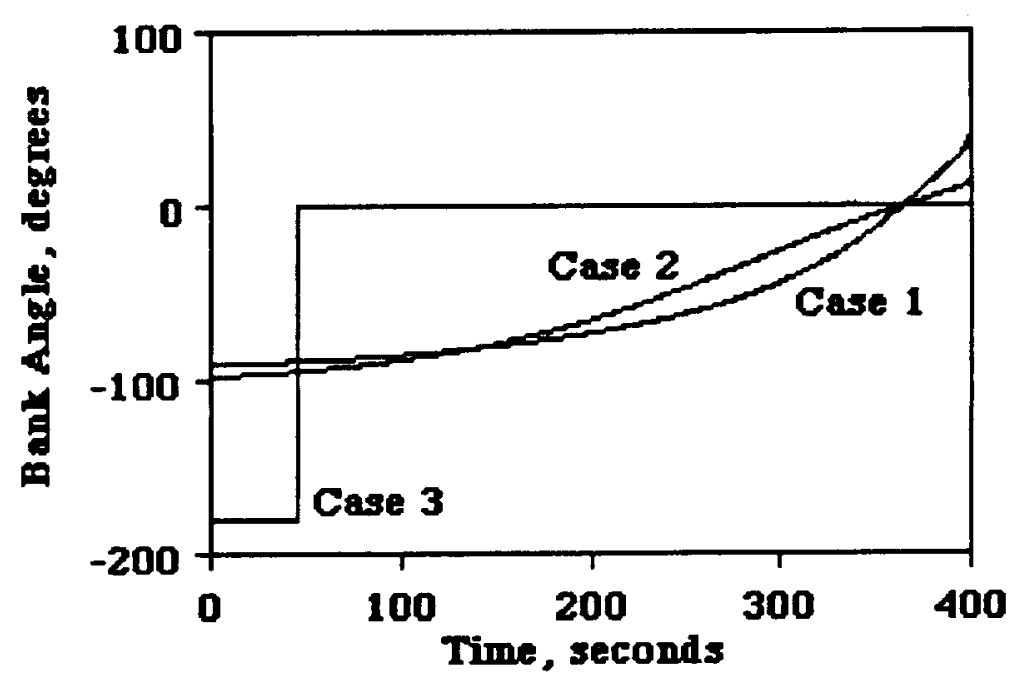

\section{Fig. 19. A Comparison of Vehicle Bank Angle Histories}

Finally, the vehicle drag modulation and thrust histories are given in Figures 20 and 21 . Both case 2 and 3 call for significantly higher thrust than Case 1 . These increased thrust magnitudes are used primarily for offsetting the increased drag caused bu flight at lower altitudes. On the other hand, both these cases use lower drag modulation than Case 1. 


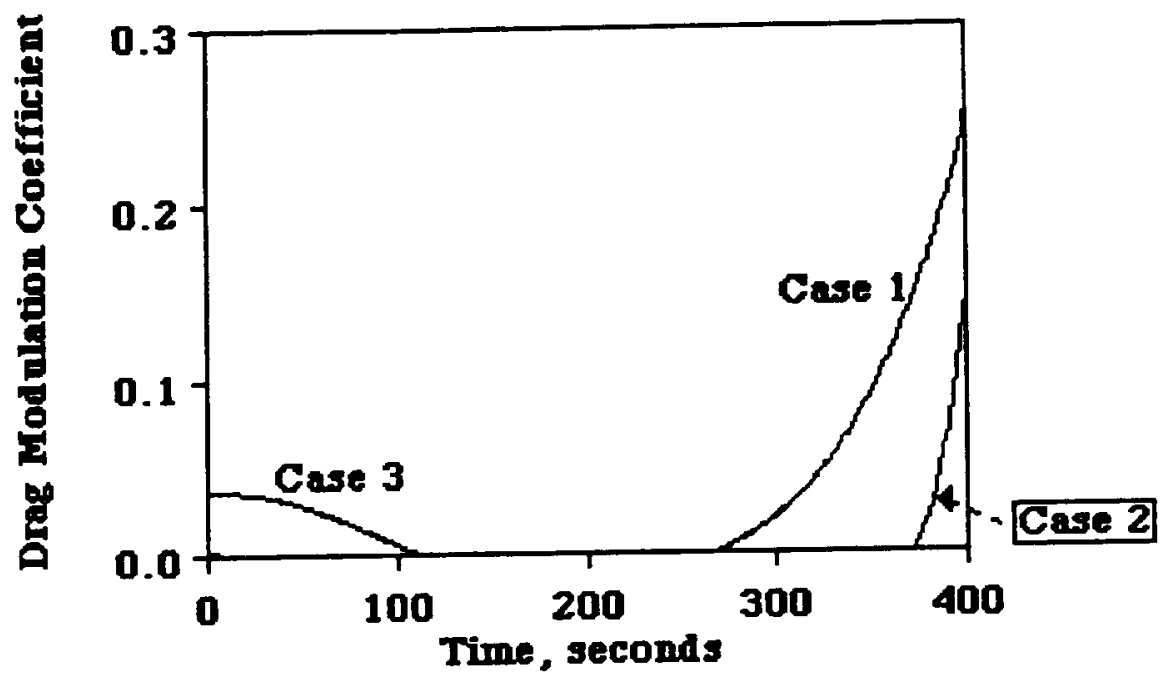

Fig. 20. Comparison of Drag Modulation Coefficients

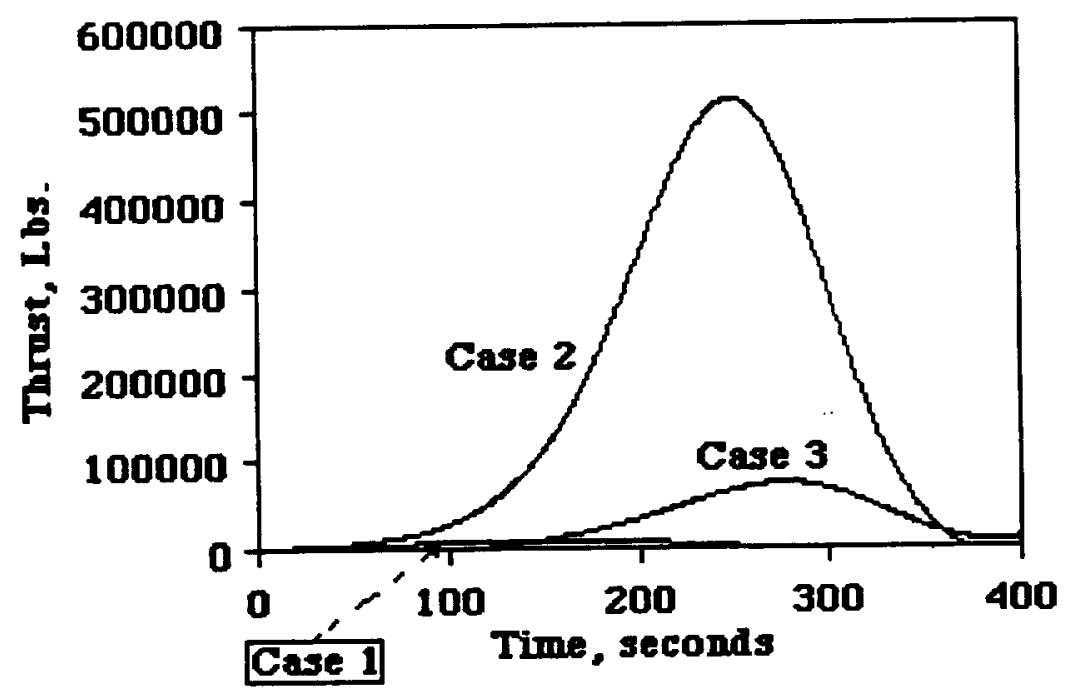

Fig. 21. A Comparison of Thrust Histories

\section{Conclusions}

This paper presented the development of a nonlinear feedback guidance law for the aero-assisted orbit transfer maneuvers. It is useful for the simultaneous change of both in-plane and out-of-plane orbital elements. The analysis employed a nonlinear point-mass model of the spacecraft. Other modeling details were the inclusion of arbitrary-order gravitational model, atmosphere rotation, ambient winds and an atmosphere density model of arbitrary complexity. 
For guidance law development, the vehicle model was first transformed into a linear, time invariant form. In order to minimize the structural load on the vehicle, the performance objective was assumed to be the minimization of the square of the vehicle acceleration. The velocity vector orientation and the position vector components at the atmosphere exit were specified. The guidance problem was solved in feedback form in the transformed coordinates. Inverse transformation of this solution produced the nonlinear guidance law for aero-assisted orbit transfer. The guidance law is in closed-loop, state feedback form and uses the vehicle performance data in the feedback loop. Numerical results using a maneuverable re-entry vehicle data were given.

\section{References}

[1] London, H. S., "Change of Satellite Orbit Plane by Aerodynamic Maneuvering", Journal of Aerospace Sciences, Vol. 29, March 1962, pp. 232-332.

[2] Miele, A., and Lee, W. Y., "Optimal Trajectories for Hypervelocity Flight", Proceedings of the 1989 American Control Conference, June 21-23, Pittsburgh, PA, pp. 2017-2023.

[3] Mease, K. D., Vinh, N. X., "Minimum-Fuel Aeroassisted Coplanar Orbit Transfer Using Lift Modulation", Journal of Guidance, Control, and Dynamics, Vol. 8, Jan. - Feb. 1985, pp. 134141.

[4] Vinh, N. X., Johannesen, J. R., Mease, K. D., Hanson, J. M., "Explicit Guidance of DragModulated Aeroassisted Transfer Between Elliptical Orbits", Journal of Guidance, Control, and Dynamics, Vol. 9, May - June 1986, pp. 274-280.

[5] Speyer, J. L., and Crues, E. Z., "Approximate Optimal Guidance Law for Aeroassisted PlaneChange Maneuvers", Journal of Guidance, Control, and Dynamics, Vol. 13, Sept. - Oct. 1990, pp. $792-802$.

[6] Calise, A. J., and Bae, G. H., "Optimal Heading Change with Minimum Energy Loss for a Hypersonic Gliding Vehicle", Journal of Guidance, Control, and Dynamics, Vol. 13, July-Aug. 1990.

[7] Miele, A., and Wang, T., "Gamma Guidance of Trajectories for Coplanar, Aeroassisted Orbit Transfer", Journal of Guidance, Control, and Dynamics, Vol. 15, Jan. - Feb. 1992, pp. 255 262. 
[8] Hull, D. G., Giltner, J. M., Speyer, J. L., and Mapar, J., "Minimum Energy-Loss Guidance for Aero-Assisted Orbital Plane Change", Journal of Guidance, Control, and Dynamics, Vol. 8, 1985, pp. 487-493.

[9] Mishne, D., Melamed, N., and Shinar, J., "Aeroassisted Transfer Between Elliptical Orbits Using Lift Control", Journal of Guidance, Control, and Dynamics, Vol. 13, Sept. - Oct. 1990, pp. $785-791$.

[10] Mease, K. D., Vinh, N. X., and Kuo, S. H., "Optimal Plane Change During Constant Altitude Hypersonic Flight", Journal of Guidance, Control, and Dynamics, July - August 1991, pp. 797 - 806.

[11] Lee, B., and Grantham, W. J., "Aeroassisted Orbital Maneuvering Using Lyapunov Optimal Feedback Control", Journal of Guidance, Control, and Dynamics, Vol. 12, March - April 1989, pp. 237 - 242.

[12] Ikawa, H., "Effect of Rotating Earth for Analysis of Aeroassisted Orbital Transfer Vehicles", Journal of Guidance, Control, and Dynamics, Vol. 11, Jan. - Feb. 1988, pp. 47 - 242.

[13] Menon, P. K. A., "Aerobrake Guidance Law Synthesis Using Feedback Linearization", 1991 American Control Conference, June 26 - 28, Boston, MA.

[14] Menon, P. K. A., and Calise, A. J., "Interception, Evasion, Rendezvous, and Velocity-to-beGained Guidance for Spacecraft", AIAA Guidance, Navigation, and Control Conference, Aug. 17-19, 1987, Monterey, CA. Paper No. AIAA-87-2318-CP.

[15] Menon, P. K. A., and Duke, E. L., "Time-Optimal Aircraft Pursuit-Evasion with a Weapon Envelope Constraint", 1990 American Control Conference, May 23-25, San Diego, CA. Also to appear in the Journal of Guidance, Control, and Dynamics.

[16] Bate, R. R., Muller, D. D., and White, J. E., Fundamentals of Astrodynamics, Dover Publications, 1971.

[17] Bryson, A. E., and Ho, Y. C., Applied Optimal Control, Hemisphere, Washington, 1975:

[18] Ewing, G. M., Calculus of Variations with Applications, Dover, New York, 1969. 
Appendix - A

\section{Implementation of the AOTV Guidance Law on a Macintosh Computer :}

All the results given in this report were generated using a point-mass simulation implemented on a Macintosh computer. In this section, some of the details of the Graphical User Interface for the AOTV simulation are given.

Four menu items at the top of these figures are used to control the program operation. The three choices available under the TRAJECTORY menu items are : Data, Run, Terminate. Selecting the Data item results in the screen display:

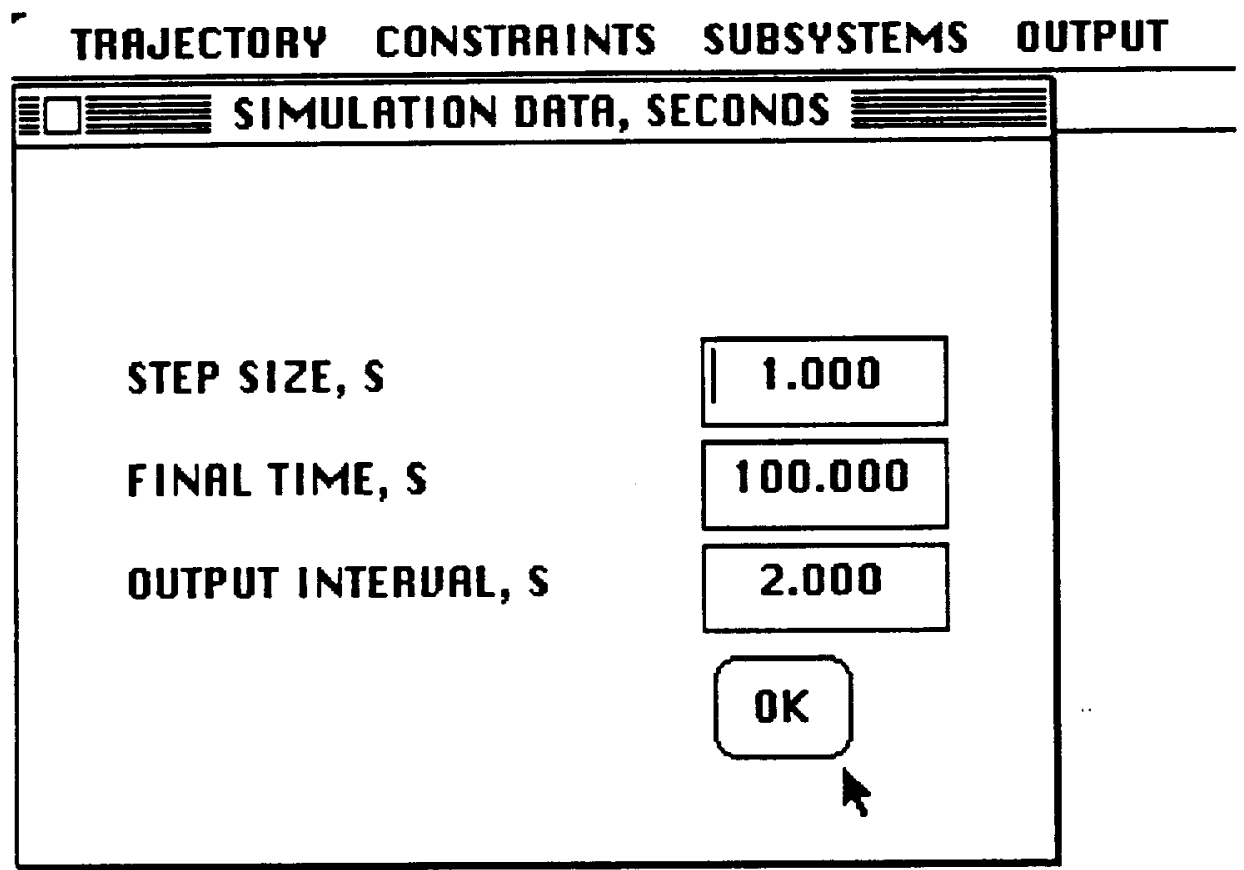

These edit fields can be used to change integration step size, final time and the output interval. The other two choices in this menu causes the simulation run or termination.

The CONSTRAINTS menu items allow for the input of boundary conditions, and timeacceleration weights in the performance index. As an illustrative example, the following illustrates the screen format for the 'boundary conditions' menu item choice. 


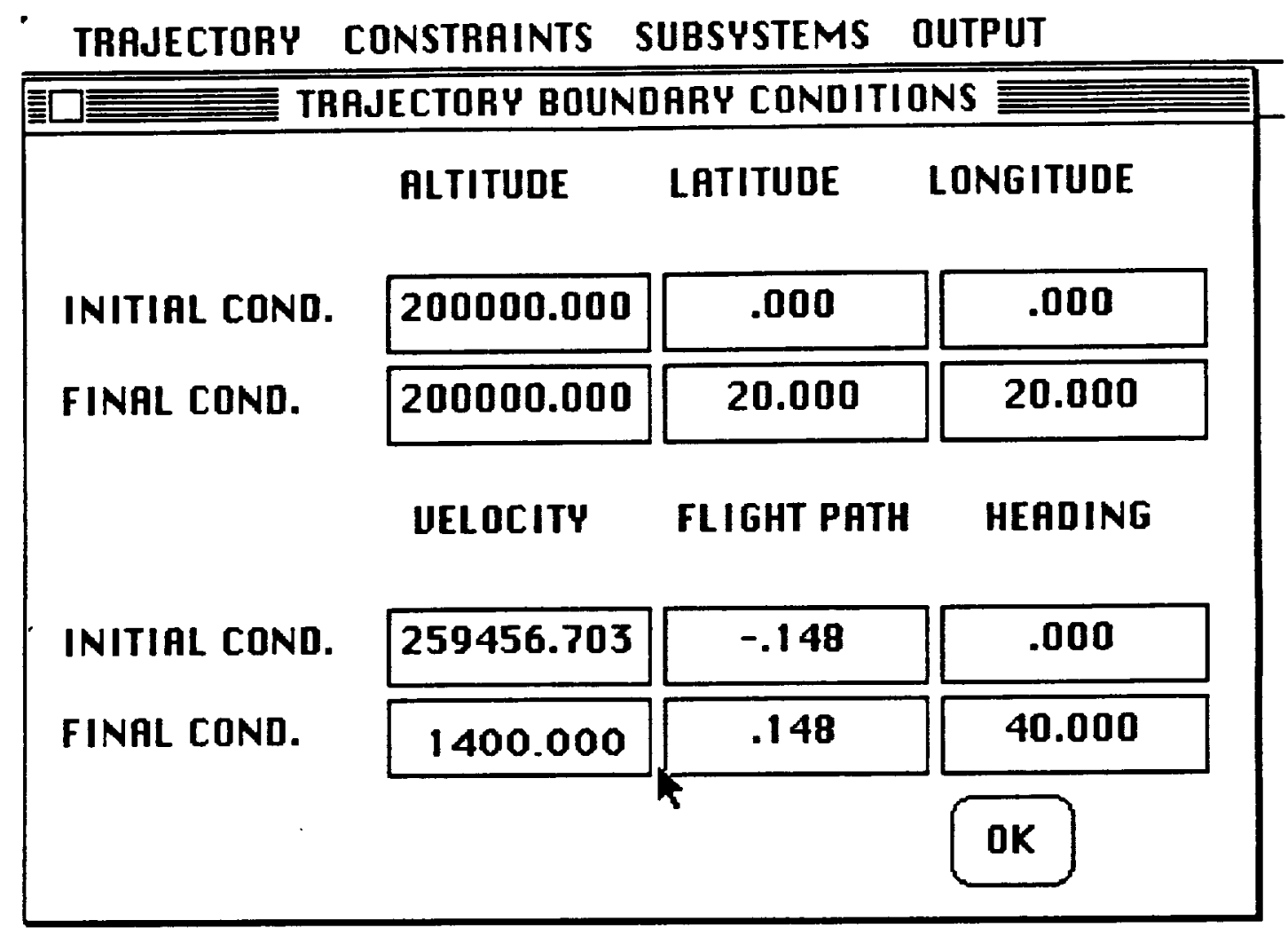

The SUBSYSTEMS menu item allows the input of model subsystem data such as: Aerodynamics, Atmosphere Density Model, Wind Profile, and Gravitation Harmonics. The screen displays for Aerodynamics, Atmosphere Density Model, Gravitation, and Wind Profile menu items are shown in the following figures. 


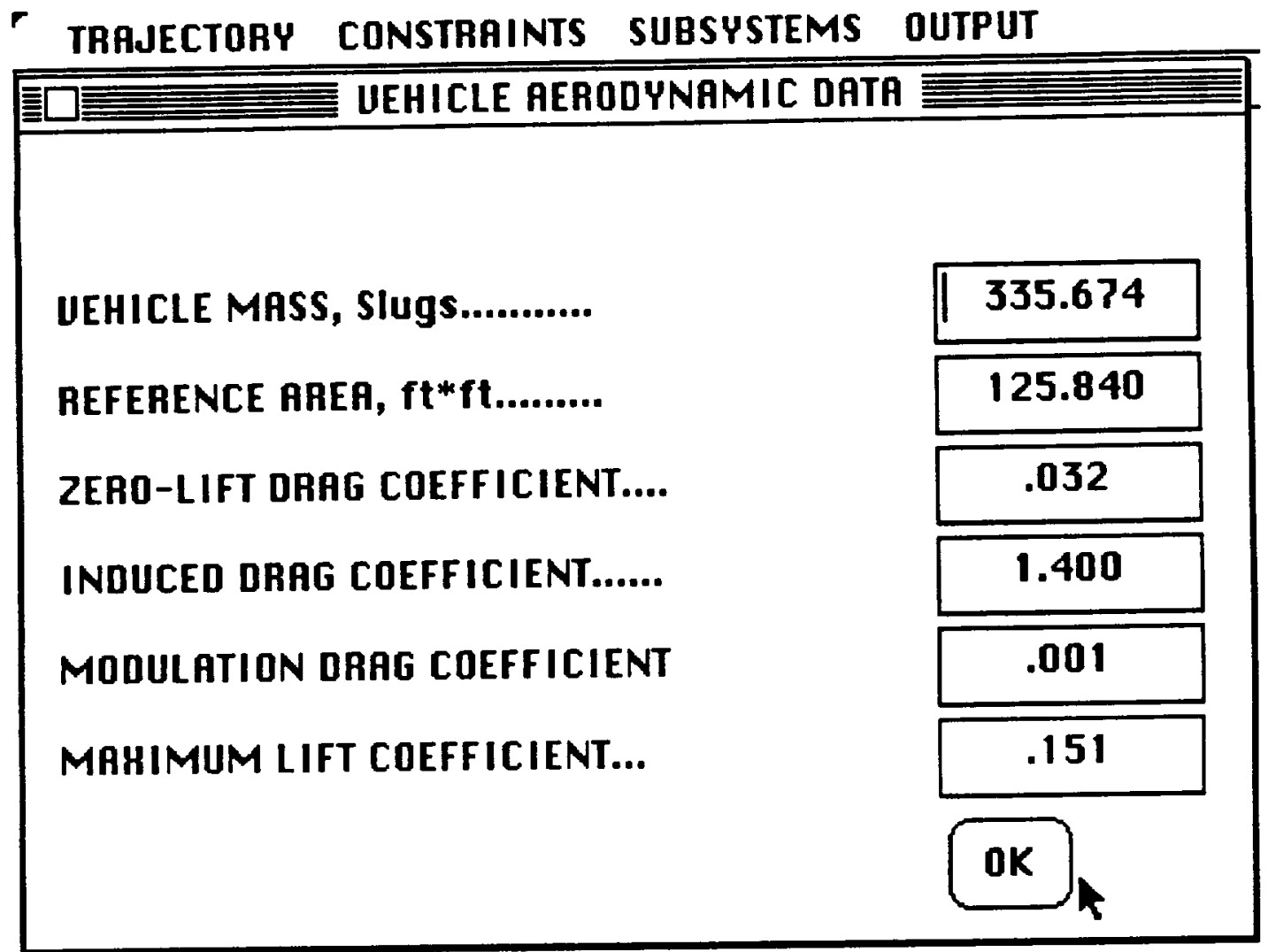

Clicking on the OK edit field will cause the program to accept the values typed in the edit fields. As currently configured, the user interface accepts data up to four decimal places. 


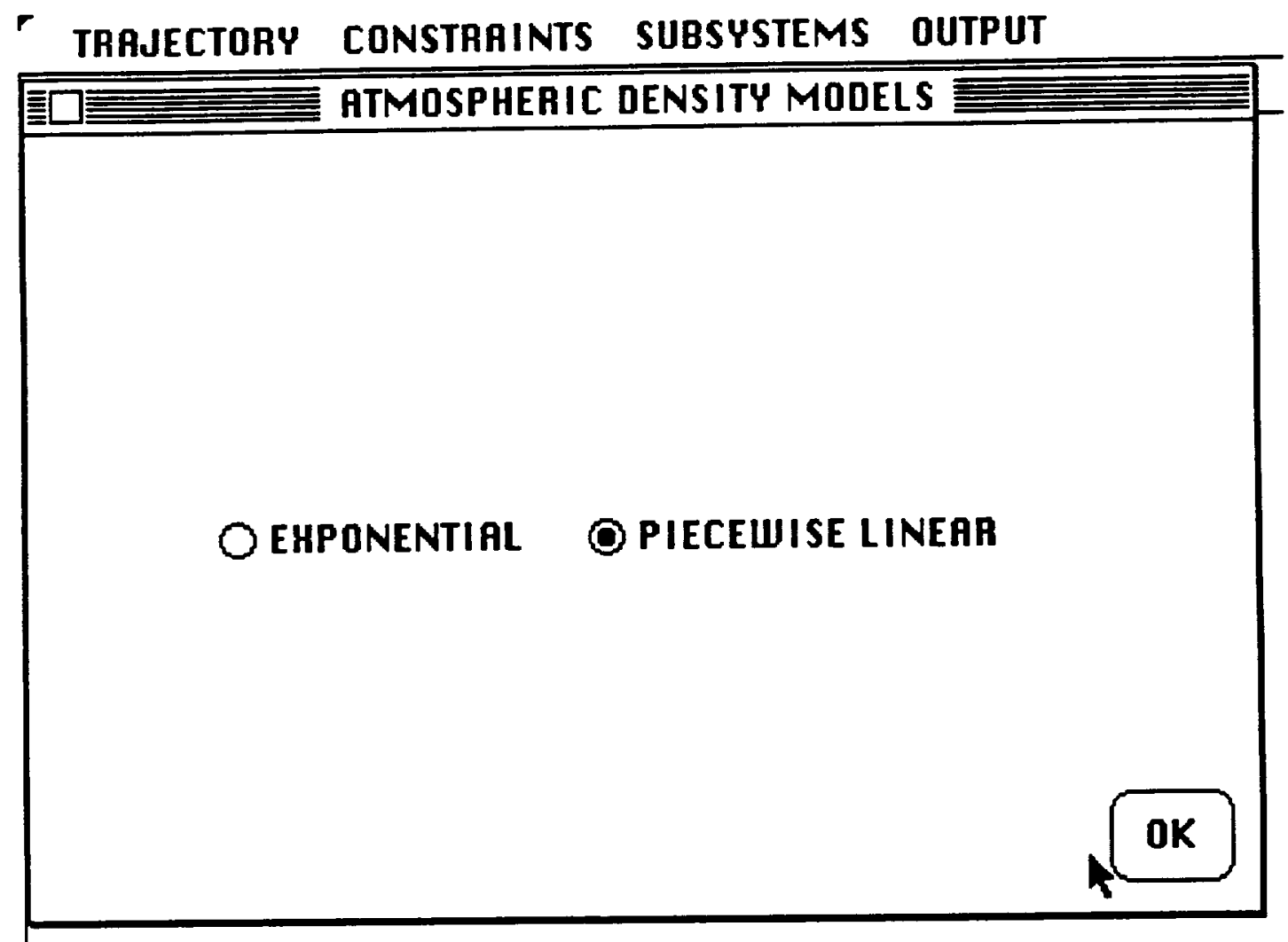

At the present time, the program permits the use of either an exponential or piecewise linear model of Earth. Future versions may include the atmospheric data for various other planets also.

Similarly, the program currently incorporates a multi-harmonic gravitational model of earth. The number of gravitational harmonics included in the formulation can be increased or decreased by clicking on 'up' or 'down' fields. Inclusion of the gravitational acceleration of other planets will be a future item. 


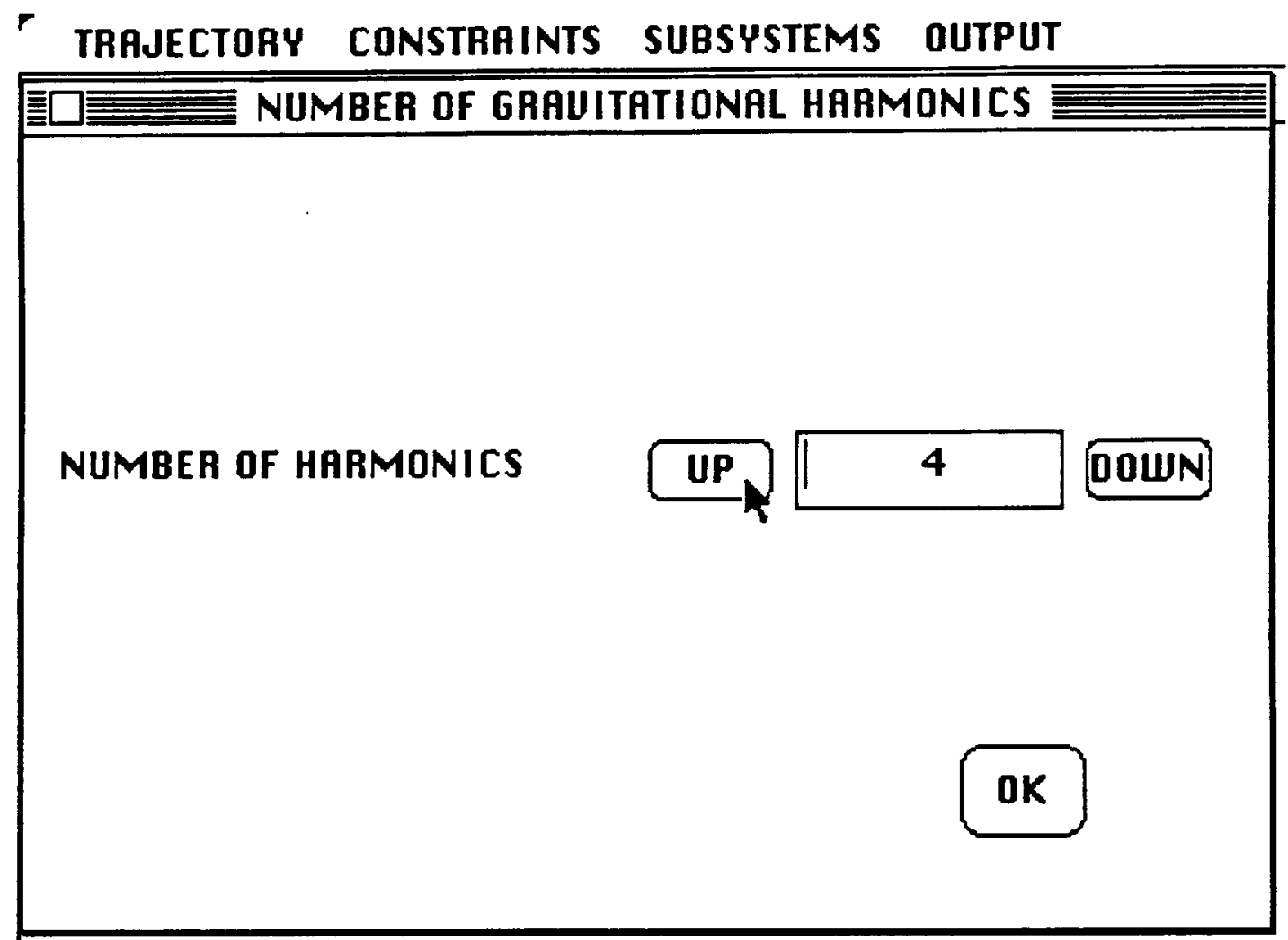

The ambient wind model currently accepts the wind velocity and its azimuth specified as a function of altitude. Inclusion of latitude-longitude dependence will be undertaken in the future. 


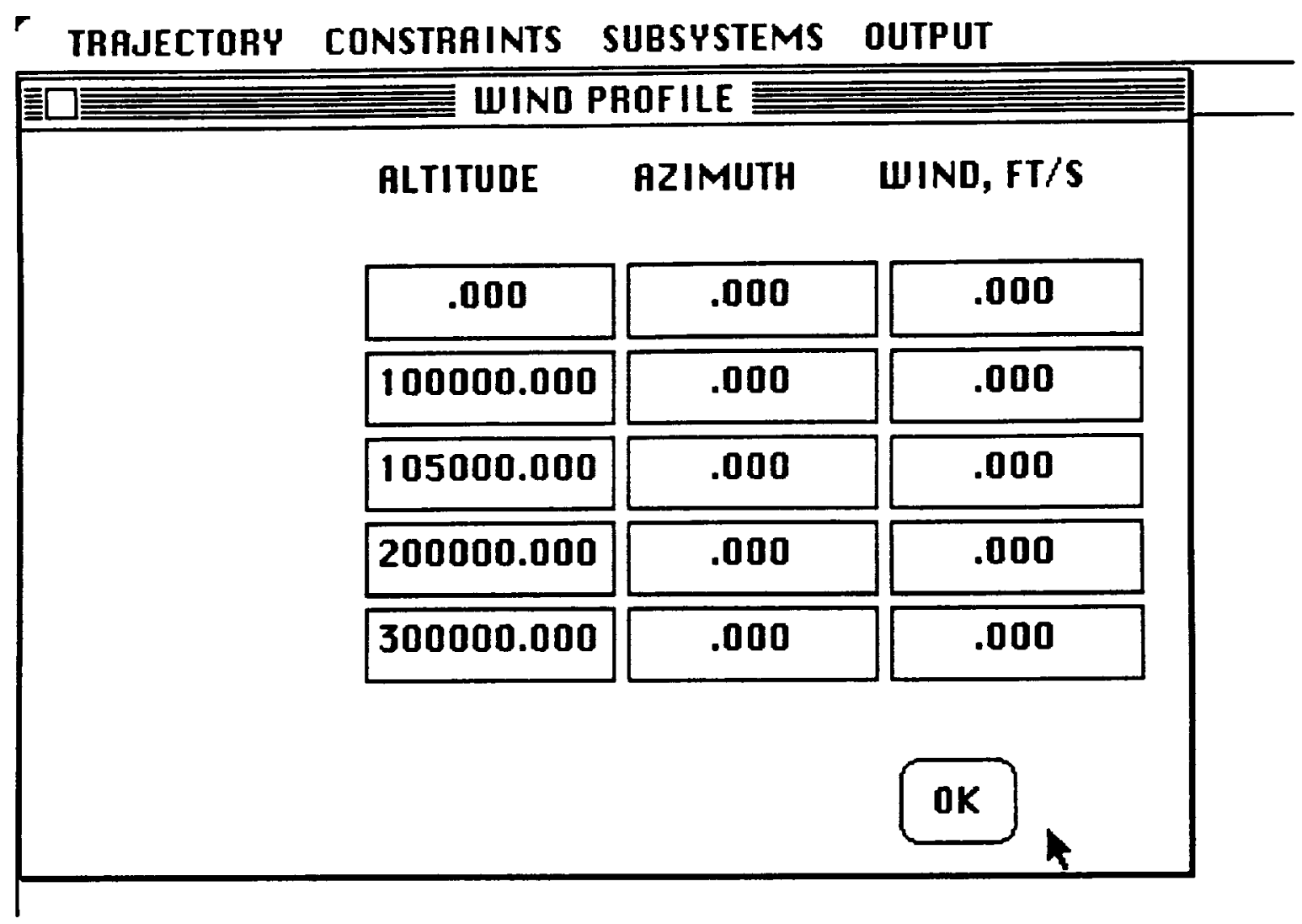

Finally, the OUTPUT menu item allows access to the simulation results, either as an output data file compatible with plotting package such as CRICKET ${ }^{\mathrm{TM}}$ or as interactive data display.

At the time of this writing, the program kernel and the graphic user interfaces have been tested in a stand alone mode. Integration and comprehensive testing of these packages will be of future interest. 